ANALYSIS \& PDE Volume 6 No. $4 \quad 2013$

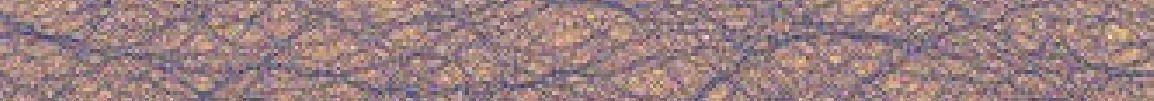
YIHU AND XIAOCHUNLI

DISCRETE FOURIER RESTRICTION ASSOCIATED WITH KDV EQUATIONS 


\title{
DISCRETE FOURIER RESTRICTION ASSOCIATED WITH KDV EQUATIONS
}

\author{
Yi Hu AND XIAOCHUN LI
}

\begin{abstract}
In this paper, we consider a discrete restriction associated with $\mathrm{KdV}$ equations. Some new Strichartz estimates are obtained. We also establish the local well-posedness for the periodic generalized Kortewegde Vries equation with nonlinear term $F(u) \partial_{x} u$ provided $F \in C^{5}$ and the initial data $\phi \in H^{s}$ with $s>1 / 2$.
\end{abstract}

\section{Introduction}

The discrete restriction problem associated with $\mathrm{KdV}$ equations is a problem asking the best constant $A_{p, N}$ satisfying

$$
\sum_{n=-N}^{N}\left|\hat{f}\left(n, n^{3}\right)\right|^{2} \leq A_{p, N}\|f\|_{p^{\prime}}^{2},
$$

where $f$ is a periodic function on $\mathbb{T}^{2}, \hat{f}$ is the Fourier transform of $f$ on $\mathbb{T}^{2}, p \geq 2$, and $p^{\prime}=p /(p-1)$. It is natural to pose a conjecture asserting that for any $\varepsilon>0, A_{p, N}$ satisfies

$$
A_{p, N} \leq \begin{cases}C_{p} N^{1-8 / p+\varepsilon} & \text { for } p \geq 8 \\ C_{p} & \text { for } 2 \leq p<8 .\end{cases}
$$

It was proved by Bourgain that $A_{6, N} \leq N^{\varepsilon}$. The desired upper bound for $A_{8, N}$ is not yet obtained; however, we are able to establish an affirmative answer for large $p$.

Theorem 1.1. Let $A_{p, N}$ be defined as in (1-1). If $p \geq 14$, for any $\varepsilon>0$, there exists a constant $C_{p}$ independent of $N$ such that

$$
A_{p, N} \leq C_{p} N^{1-8 / p+\varepsilon} .
$$

The periodic Strichartz inequality associated to KdV equations is the inequality seeking the best constant $K_{p, N}$ satisfying

$$
\left\|\sum_{n=-N}^{N} a_{n} e^{2 \pi i t n^{3}+2 \pi i x n}\right\|_{L_{x, t}^{p}(\mathbb{T} \times \mathbb{T})} \leq K_{p, N}\left(\sum_{n=-N}^{N}\left|a_{n}\right|^{2}\right)^{1 / 2} .
$$

By duality, we immediately see that

$$
K_{p, N} \sim \sqrt{A_{p, N}} .
$$

This work was partially supported by NSF grant DMS-0801154.

MSC2010: primary 35G25; secondary 42A16.

Keywords: discrete Fourier restriction, KdV, local well-posedness. 
Hence, Theorem 1.1 is equivalent to Strichartz estimates,

$$
K_{p, N} \leq C N^{1 / 2-4 / p+\varepsilon}, \quad \text { for } p \geq 14
$$

It was observed by Bourgain that the periodic Strichartz inequalities (1-4) for $p=4,6$ are crucial for obtaining the local well-posedness of periodic $\mathrm{KdV}(\mathrm{mKdV}$ or $\mathrm{gKdV})$. The local (global) well-posedness of periodic KdV for $s \geq 0$ was first studied by Bourgain [1993b]. Via a bilinear estimate approach, Kenig, Ponce, and Vega [Kenig et al. 1996] established the local well-posedness of periodic KdV for $s>-1 / 2$. The sharp global well-posedness of the periodic KdV was proved by Colliander, Keel, Staffilani, Takaoka, and Tao [Colliander et al. 2003], by utilizing the $I$-method.

Inspired by Bourgain's work, we can obtain the following theorem on $\mathrm{gKdV}$. Here the $\mathrm{gKdV}$ is the generalized Korteweg-de Vries (gKdV) equation

$$
\left\{\begin{array}{l}
u_{t}+u_{x x x}+u^{k} u_{x}=0, \\
u(x, 0)=\phi(x), \quad x \in \mathbb{T}, t \in \mathbb{R},
\end{array}\right.
$$

where $k \in \mathbb{N}$ and $k \geq 3$.

Theorem 1.2. The Cauchy problem (1-6) is locally well-posed if the initial data $\phi \in H^{s}$ for $s>1 / 2$.

Theorem 1.2 is not new. It was proved by Colliander, Keel, Staffilani, Takaoka, and Tao [Colliander et al. 2004], but our method is different. The method used by those authors is based on a rescaling argument and the bilinear estimates proved by Kenig, Ponce and Vega [Kenig et al. 1996]. Our method is more straightforward and does not need the rescaling argument, the bilinear estimates, or the multilinear estimates in the earlier papers. This allows us to extend Theorem 1.2 to a very general setting. More precisely, consider the Cauchy problem for periodic generalized Korteweg-de Vries (gKdV) equation

$$
\left\{\begin{array}{l}
u_{t}+u_{x x x}+F(u) u_{x}=0, \\
u(x, 0)=\phi(x), \quad x \in \mathbb{T}, t \in \mathbb{R} .
\end{array}\right.
$$

Here $F$ is a suitable function. Then the following theorem can be established.

Theorem 1.3. The Cauchy problem (1-7) is locally well-posed provided $F$ is a $C^{5}$ function and the initial data $\phi \in H^{s}$ for $s>1 / 2$.

For sufficiently smooth $F$, say $F \in C^{15}$, the existence of a local solution of (1-7) for $s \geq 1$ and the global well-posedness of (1-7) for small data $\phi \in H^{s}$ with $s>3 / 2$ were proved by Bourgain [1995]. The index $1 / 2$ is sharp because the ill-posedness of (1-6) for $s<1 / 2$ is known; see [Colliander et al. 2004]. In order to make Theorem 1.3 well-posed for the initial data $\phi \in H^{s}$ with $s>1 / 2$, the sharp regularity condition for $F$ is perhaps $C^{4}$. But the method utilized in this paper, with a small modification, seems only to be able to reach an affirmative result for $F \in C^{(9 / 2)+}$ and $s>1 / 2$. Moreover, the endpoint $s=1 / 2$ case could possibly be done by combining the ideas from [Colliander et al. 2004] and this paper. We do not pursue this here. 


\section{Proof of Theorem 1.1}

Proof. To prove Theorem 1.1, we need to introduce a level set. Since $\sqrt{A_{p, N}} \sim K_{p, N}$, it suffices to prove the Strichartz estimates (1-4). Let $F_{N}$ be a periodic function on $\mathbb{T}^{2}$ given by

$$
F_{N}(x, t)=\sum_{n=-N}^{N} a_{n} e^{2 \pi i n x} e^{2 \pi i n^{3} t}
$$

where $\left\{a_{n}\right\}$ is a sequence with $\sum_{n}\left|a_{n}\right|^{2}=1$ and $(x, t) \in \mathbb{T}^{2}$. For any $\lambda>0$, set a level set $E_{\lambda}$ to be

$$
E_{\lambda}=\left\{(x, t) \in \mathbb{T}^{2}:\left|F_{N}(x, t)\right|>\lambda\right\} .
$$

To obtain the desired estimate for the level set, let us first state a lemma on Weyl's sums.

Lemma 2.1. Suppose that $t \in \mathbb{T}$ satisfies $|t-a / q| \leq 1 / q^{2}$, where $a$ and $q$ are relatively prime. Then if $q \geq N^{2}$

$$
\left|\sum_{n=1}^{N} e^{2 \pi i\left(t n^{3}+b n^{2}+c n\right)}\right| \leq C N^{1 / 4+\varepsilon} q^{1 / 4} .
$$

Here $b$ and $c$ are real numbers, and the constant $C$ is independent of $b, c, t, a, q$, and $N$.

The proof of Lemma 2.1 relies on Weyl's squaring method. See [Hua 1965] or [Montgomery 1994] for details. We also need the following lemma.

Lemma 2.2 [Bourgain 1993a]. For any integer $Q \geq 1$ and any integer $n \neq 0$, and any $\varepsilon>0$,

$$
\sum_{Q \leq q<2 Q}\left|\sum_{a \in \mathscr{P}_{q}} e^{2 \pi i(a / q) n}\right| \leq C_{\varepsilon} d(n, Q) Q^{1+\varepsilon} .
$$

Here $\mathscr{P}_{q}$ is given by

$$
\mathscr{P}_{q}=\{a \in \mathbb{N}: 1 \leq a \leq q \text { and }(a, q)=1\},
$$

and $d(n, Q)$ denotes the number of divisors of $n$ less than $Q$ and $C_{\varepsilon}$ is a constant independent of $Q, n$.

Lemma 2.2 can be proved by observing that the arithmetic function defined by $f(q)=\sum_{a \in \mathscr{P}_{q}} e^{2 \pi i(a / q) n}$ is multiplicative, and then utilizing the prime factorization for $q$ to conclude the lemma.

Proposition 2.3. Let $K_{N}$ be a kernel defined by

$$
K_{N}(x, t)=\sum_{n=-N}^{N} e^{2 \pi i t n^{3}+2 \pi i x n} .
$$

For any given positive number $Q$ with $N^{2} \leq Q \leq N^{3}$, the kernel $K_{N}$ can be decomposed into $K_{1, Q}+K_{2, Q}$ such that

$$
\left\|K_{1, Q}\right\|_{\infty} \leq C_{1} N^{1 / 4+\varepsilon} Q^{1 / 4} .
$$

and

$$
\left\|\widehat{K_{2, Q}}\right\|_{\infty} \leq \frac{C_{2} N^{\varepsilon}}{Q}
$$


Here the constants $C_{1}, C_{2}$ are independent of $Q$ and $N$.

Proof. We can assume that $Q$ is an integer, since otherwise we can take the integer part of $Q$. For a standard bump function $\varphi$ supported on [1/200,1/100], we set

$$
\Phi(t)=\sum_{Q \leq q \leq 5 Q} \sum_{a \in \mathscr{P}_{q}} \varphi\left(\frac{t-a / q}{1 / q^{2}}\right) .
$$

Clearly $\Phi$ is supported on $[0,1]$. We can extend $\Phi$ to other intervals periodically to obtain a periodic function on $\mathbb{T}$. This periodic function, generated by $\Phi$, will also be denoted by $\Phi$. It is easy to see that

$$
\widehat{\Phi}(0)=\sum_{q \sim Q} \sum_{a \in \mathscr{P}_{q}} \frac{\mathscr{F}_{\mathbb{R}} \varphi(0)}{q^{2}}=\sum_{q \sim Q} \frac{\phi(q)}{q^{2}} \mathscr{F}_{\mathbb{R}} \varphi(0)
$$

is a constant independent of $Q$. Here $\phi$ is Euler's phi function, and $\mathscr{F}_{\mathbb{R}}$ denotes the Fourier transform of a function on $\mathbb{R}$. Also we have

$$
\widehat{\Phi}(k)=\sum_{q \sim Q} \sum_{a \in \mathscr{P}_{q}} \frac{1}{q^{2}} e^{-2 \pi i(a / q) k \mathscr{F}_{\mathbb{R}} \varphi\left(k / q^{2}\right)} .
$$

Applying Lemma 2.2 and the fact that $Q \leq N^{3}$, we obtain

$$
|\widehat{\Phi}(k)| \leq \frac{N^{\varepsilon}}{Q},
$$

if $k \neq 0$.

We now define

$$
K_{1, Q}(x, t)=\frac{1}{\widehat{\Phi}(0)} K_{N}(x, t) \Phi(t) \quad \text { and } \quad K_{2, Q}=K_{N}-K_{1, Q} .
$$

Equation (2-6) follows from Lemma 2.1 since the intervals $J_{a / q}=\left[\frac{a}{q}+\frac{1}{100 q^{2}}, \frac{a}{q}+\frac{1}{50 q^{2}}\right]$ are pairwise disjoint for all $Q \leq q \leq 5 Q$ and $a \in \mathscr{P}_{q}$.

We now prove (2-7). In fact, represent $\Phi$ as its Fourier series to get

$$
K_{2, Q}(x, t)=-\frac{1}{\widehat{\Phi}(0)} \sum_{k \neq 0} \widehat{\Phi}(k) e^{2 \pi i k t} K_{N}(x, t) .
$$

Thus its Fourier coefficient is

$$
\widehat{K_{2, Q}}\left(n_{1}, n_{2}\right)=-\frac{1}{\widehat{\Phi}(0)} \sum_{k \neq 0} \widehat{\Phi}(k) \mathbf{1}_{\left\{n_{2}=n_{1}^{3}+k\right\}}(k) .
$$

Here $\left(n_{1}, n_{2}\right) \in \mathbb{Z}^{2}$ and $\mathbf{1}_{A}$ is the indicator function of a set $A$. This implies that $\widehat{K_{2, Q}}\left(n_{1}, n_{2}\right)=0$ if $n_{2}=n_{1}^{3}$, and if $n_{2} \neq n_{1}^{3}$,

$$
\widehat{K_{2, Q}}\left(n_{1}, n_{2}\right)=-\frac{1}{\widehat{\Phi}(0)} \widehat{\Phi}\left(n_{2}-n_{1}^{3}\right)
$$


Applying (2-11), we estimate $\widehat{K_{2, Q}}\left(n_{1}, n_{2}\right)$ by

$$
\left|\widehat{K_{2, Q}}\left(n_{1}, n_{2}\right)\right| \leq \frac{C N^{\varepsilon}}{Q},
$$

since $N \leq Q \leq N^{2}$. Hence we obtain (2-7), completing the proof.

Now we can state our theorem on the level set estimates.

Theorem 2.4. For any positive numbers $\varepsilon$ and $Q \geq N^{2}$, the level set defined as in (2-2) satisfies

$$
\lambda^{2}\left|E_{\lambda}\right|^{2} \leq C_{1} N^{1 / 4+\varepsilon} Q^{1 / 4}\left|E_{\lambda}\right|^{2}+\frac{C_{2} N^{\varepsilon}}{Q}\left|E_{\lambda}\right|
$$

for all $\lambda>0$. Here $C_{1}$ and $C_{2}$ are constants independent of $N$ and $Q$.

Proof. Notice that if $Q \geq N^{3}$, (2-12) becomes trivial, since $E_{\lambda}=\varnothing$ if $\lambda \geq C N^{1 / 2}$. So we can assume that $N^{2} \leq Q \leq N^{3}$. For the function $F_{N}$ and the level set $E_{\lambda}$ given in (2-1) and (2-2), respectively, we define $f$ to be

$$
f(x, t)=\frac{F_{N}(x, t)}{\left|F_{N}(x, t)\right|} \mathbf{1}_{E_{\lambda}}(x, t) .
$$

Clearly

$$
\lambda\left|E_{\lambda}\right| \leq \int_{\mathbb{T}^{2}} \overline{F_{N}(x, t)} f(x, t) d x d t .
$$

By the definition of $F_{N}$, we get

$$
\lambda\left|E_{\lambda}\right| \leq \sum_{n=-N}^{N} \overline{a_{n}} \hat{f}\left(n, n^{3}\right) .
$$

Utilizing the Cauchy-Schwarz inequality, we have

$$
\lambda^{2}\left|E_{\lambda}\right|^{2} \leq \sum_{n=-N}^{N}\left|\hat{f}\left(n, n^{3}\right)\right|^{2} .
$$

The right hand side can be written as

$$
\left\langle K_{N} * f, f\right\rangle .
$$

For any $Q$ with $N^{2} \leq Q \leq N^{3}$, we employ Proposition 2.3 to decompose the kernel $K_{N}$. We then have

$$
\lambda^{2}\left|E_{\lambda}\right|^{2} \leq\left|\left\langle K_{1, Q} * f, f\right\rangle\right|+\left|\left\langle K_{2, Q} * f, f\right\rangle\right| .
$$

From (2-6) and (2-7), we then obtain

$$
\lambda^{2}\left|E_{\lambda}\right|^{2} \leq C_{1} N^{1 / 4+\varepsilon} Q^{1 / 4}\|f\|_{1}^{2}+\frac{C_{2} N^{\varepsilon}}{Q}\|f\|_{2}^{2} \leq C_{1} N^{1 / 4+\varepsilon} Q^{1 / 4}\left|E_{\lambda}\right|^{2}+\frac{C_{2} N^{\varepsilon}}{Q}\left|E_{\lambda}\right| .
$$

Corollary 2.5. If $\lambda \geq 2 C_{1} N^{3 / 8+\varepsilon,}$

$$
\left|E_{\lambda}\right| \leq \frac{C N^{1+\varepsilon}}{\lambda^{10}}
$$

Here $C_{1}$ is the constant $C_{1}$ in Theorem 2.4 and $C$ is a constant independent of $N$ and $\lambda$. 
Proof. Since $\lambda \geq 2 C_{1} N^{3 / 8+\varepsilon}$, we simply take $Q$ satisfying $2 C_{1} N^{1 / 4+\varepsilon} Q^{1 / 4}=\lambda^{2}$. Then Corollary 2.5 follows from Theorem 2.4 .

We are now ready to finish the proof of Theorem 1.1. In fact, let $p \geq 14$ and write $\|F\|_{p}^{p}$ as

$$
p \int_{0}^{2 C_{1} N^{3 / 8+\varepsilon}} \lambda^{p-1}\left|E_{\lambda}\right| d \lambda+p \int_{2 C_{1} N^{3 / 8+\varepsilon}}^{2 N^{1 / 2}} \lambda^{p-1}\left|E_{\lambda}\right| d \lambda .
$$

Observe that $A_{6, N} \leq N^{\varepsilon}$ implies

$$
\left|E_{\lambda}\right| \leq \frac{N^{\varepsilon}}{\lambda^{6}} .
$$

Thus the first term in (2-16) is bounded by

$$
C N^{3(p-6) / 8+\varepsilon} \leq C N^{p / 2-4+\varepsilon},
$$

since $p \geq 14$. From (2-15), the second term is majorized by

$$
C N^{p / 2-4+\varepsilon} \text {. }
$$

Putting both estimates together, we complete the proof of Theorem 1.1.

\section{A Lower bound of $A_{p, N}$}

In this section we show that $N^{1-8 / p}$ is the best upper bound of $A_{p, N}$ if $p \geq 8$. Hence (1-3) can not be improved substantially, and it is sharp up to a factor of $N^{\varepsilon}$.

For $b \in \mathbb{N}$, let $J(N ; b)$ be defined by

$$
S(N ; b)=\int_{\mathbb{T}^{2}}\left|\sum_{n=-N}^{N} e^{2 \pi i t n^{3}+2 \pi i x n}\right|^{2 b} d x d t .
$$

Proposition 3.1. Let $S(N ; b)$ be defined as in (3-1). Then

$$
S(N ; b) \geq C\left(N^{b}+N^{2 b-4}\right) .
$$

Here $C$ is a constant independent of $N$.

Proof. Clearly $S(N ; b)$ is equal to the number of solutions of

$$
\left\{\begin{array}{l}
n_{1}+\cdots+n_{b}=m_{1}+\cdots+m_{b}, \\
n_{1}^{3}+\cdots+n_{b}^{3}=m_{1}^{3}+\cdots+m_{b}^{3},
\end{array}\right.
$$

with $n_{j}, m_{j} \in\{-N, \ldots, N\}$ for all $j \in\{1, \ldots, b\}$. For each $\left(m_{1}, \ldots, m_{b}\right)$, we may obtain a solution of (3-3) by taking $\left(n_{1}, \ldots, n_{b}\right)=\left(m_{1}, \ldots, m_{b}\right)$. Thus

$$
S(N ; b) \geq N^{b} .
$$

To derive a further lower bound for $S(N ; b)$, we set $\Omega$ to be

$$
\Omega=\left\{(x, t):|x| \leq \frac{1}{60 N},|t| \leq \frac{1}{60 N^{3}}\right\} .
$$


If $(x, t) \in \Omega$ and $|n| \leq N$,

$$
\left|t n^{3}+x n\right| \leq \frac{1}{30}
$$

Hence, if $(x, t) \in \Omega$,

$$
\left|\sum_{n=-N}^{N} e^{2 \pi i t n^{3}+2 \pi i x n}\right| \geq\left|\operatorname{Re} \sum_{n=-N}^{N} e^{2 \pi i t n^{3}+2 \pi i x n}\right| \geq \sum_{n=-N}^{N} \cos \left(2 \pi\left(t n^{3}+x n\right)\right) \geq C N .
$$

Consequently, we have

$$
S(N ; b) \geq \int_{\Omega}\left|\sum_{n=-N}^{N} e^{2 \pi i t n^{3}+2 \pi i x n}\right|^{2 b} d x d t \geq C N^{2 b}|\Omega| \geq C N^{2 b-4} .
$$

Proposition 3.2. Let $p \geq 2$ be even. Then $A_{p, N}$ satisfies

$$
A_{p, N} \geq C\left(1+N^{1-8 / p}\right) .
$$

Here $C$ is a constant independent of $N$.

Proof. Let $p=2 b$ since $p$ is even. Setting $a_{n}=1$ for all $n$ in the definition of $K_{p, N}$, we get

$$
S(N ; b) \leq K_{p, N}^{p}(2 N)^{b} .
$$

By Proposition 3.1, we have

$$
K_{p, N} \geq C\left(1+N^{1 / 2-4 / p}\right) .
$$

Consequently, we conclude (3-8) since $A_{p, N} \sim K_{p, N}^{2}$.

\section{An estimate of Hua}

The following theorem was proved by Hua [1965] by an arithmetic argument. We provide a different proof.

Theorem 4.1. Let $S(N ; b)$ be defined as in (3-1). Then

$$
S(N ; 5) \leq C N^{6+\varepsilon} .
$$

By Proposition 3.1, we see that the estimate (4-1) is (almost) sharp. $S(N ; 4) \leq N^{4+\varepsilon}$ is still open. Proof of Theorem 4.1. Let $G_{\lambda}$ be the level set given by

$$
G_{\lambda}=\left\{(x, t) \in \mathbb{T}^{2}:\left|K_{N}(x, t)\right| \geq \lambda\right\} .
$$

Here $K_{N}$ is the function defined as in (2-5).

Letting $f=\mathbf{1}_{G_{\lambda}} K_{N} /\left|K_{N}\right|$, we have

$$
\lambda\left|G_{\lambda}\right| \leq \sum_{n=-N}^{N} \hat{f}\left(n, n^{3}\right)=\left\langle f_{N}, K_{N}\right\rangle
$$


where $f_{N}$ is a rectangular Fourier partial sum defined by

$$
f_{N}(x, t)=\sum_{\substack{\left|n_{1}\right| \leq N \\\left|n_{2}\right| \leq N^{3}}} \hat{f}\left(n_{1}, n_{2}\right) e^{2 \pi n_{1} x} e^{2 \pi i n_{2} t} .
$$

Employing Proposition 2.3 for $K_{N}$, we estimate the level set $G_{\lambda}$ by

$$
\lambda\left|G_{\lambda}\right| \leq\left|\left\langle f_{N}, K_{1, Q}\right\rangle\right|+\left|\left\langle f_{N}, K_{2, Q}\right\rangle\right|
$$

for any $Q \geq N^{2}$. From (2-6) and (2-7), $\lambda\left|G_{\lambda}\right|$ can be bounded further by

$$
C\left(N^{1 / 4+\varepsilon} Q^{1 / 4}\left\|f_{N}\right\|_{1}+\sum_{\substack{\left|n_{1}\right| \leq N \\\left|n_{2}\right| \leq N^{3}}}\left|\widehat{K_{2, Q}}\left(n_{1}, n_{2}\right) \hat{f}\left(n_{1}, n_{2}\right)\right|\right)
$$

Thus, from the fact that the $L^{1}$ norm of Dirichlet kernel $D_{N}$ is comparable to $\log N,(2-7)$, and the Cauchy-Schwarz inequality, we have

$$
\lambda\left|G_{\lambda}\right| \leq C N^{1 / 4+\varepsilon} Q^{1 / 4}\left|G_{\lambda}\right|+\frac{C N^{2+\varepsilon}}{Q}\left|G_{\lambda}\right|^{1 / 2},
$$

for all $Q \geq N^{2}$. For $\lambda \geq 2 C N^{3 / 4+\varepsilon}$, take $Q$ to be a number satisfying

$$
2 C N^{1 / 4+\varepsilon} Q^{1 / 4}=\lambda
$$

and obtain

$$
\left|G_{\lambda}\right| \leq \frac{C N^{6+\varepsilon}}{\lambda^{10}}
$$

Notice that

$$
\left\|K_{N}\right\|_{6} \leq N^{1 / 2} K_{6, p} \leq N^{1 / 2+\varepsilon} .
$$

Hence, by (4-3), we majorize $\left|G_{\lambda}\right|$ by

$$
\left|G_{\lambda}\right| \leq \frac{C N^{3+\varepsilon}}{\lambda^{6}}
$$

We now estimate $S(N ; 5)$ by

$$
S(N ; 5) \leq C \int_{2 C N^{3 / 4+\varepsilon}}^{2 N} \lambda^{10-1}\left|G_{\lambda}\right| d \lambda+C \int_{0}^{2 C N^{3 / 4+\varepsilon}} \lambda^{10-1}\left|G_{\lambda}\right| d \lambda .
$$

From (4-8), the first term in the right hand side of (4-11) can be bounded by $C N^{6+\varepsilon}$. From (4-10), the second term is clearly bounded by $N^{6+\varepsilon}$. Putting both estimates together,

$$
S(N ; 5) \leq C N^{6+\varepsilon}
$$

as desired. 


\section{Estimates for the nonlinear term and Local well-posedness of (1-6)}

For any integrable function $u$ on $\mathbb{T} \times \mathbb{R}$, we define the space-time Fourier transform by

$$
\hat{u}(n, \lambda)=\int_{\mathbb{R}} \int_{\mathbb{T}} u(x, t) e^{-i n x} e^{-i \lambda t} d x d t
$$

and set

$$
\langle x\rangle:=1+|x| .
$$

We now introduce the $X_{s, b}$ space, initially used by Bourgain.

Definition 5.1. Let $I$ be a time interval in $\mathbb{R}$ and $s, b \in \mathbb{R}$. Let $X_{s, b}(I)$ be the space of functions $u$ on $\mathbb{} \mathbb{} \times I$ that may be represented as

$$
u(x, t)=\sum_{n \in \mathbb{Z}} \int_{\mathbb{R}} \hat{u}(n, \lambda) e^{i n x} e^{i \lambda t} d \lambda \quad \text { for }(x, t) \in \mathbb{T} \times I
$$

with the space-time Fourier transform $\hat{u}$ satisfying

$$
\|u\|_{X_{s, b}(I)}=\left(\sum_{n} \int\langle n\rangle^{2 s}\left\langle\lambda-n^{3}\right\rangle^{2 b}|\hat{u}(n, \lambda)|^{2} d \lambda\right)^{1 / 2}<\infty .
$$

Here the norm should be understood as a restriction norm.

We take the time interval to be $[0, \delta]$ for a small positive number $\delta$ and abbreviate $\|u\|_{X_{s, b}(I)}$ as $\|u\|_{s, b}$ for any function $u$ restricted to $\mathbb{T} \times[0, \delta]$. In this section, we always restrict the function $u$ to $\mathbb{T} \times[0, \delta]$. Let $w$ be the nonlinear function defined by

$$
w=\left(u^{k}-\int u^{k} d x\right) u_{x}
$$

We also define

$$
\|u\|_{Y_{s}}:=\|u\|_{s, 1 / 2}+\left(\sum_{n}\langle n\rangle^{2 s}\left(\int|\hat{u}(n, \lambda)| d \lambda\right)^{2}\right)^{1 / 2} .
$$

We need the following estimate on the nonlinear function $w$, in order to establish a contraction on the space $\left\{u:\|u\|_{Y_{s}} \leq M\right\}$ for some $M>0$.

Proposition 5.2. For $s>1 / 2$, there exists $\theta>0$ such that, for the nonlinear function $w$ given by (5-4),

$$
\|w\|_{s,-1 / 2}+\left(\sum_{n}\langle n\rangle^{2 s}\left(\int \frac{|\widehat{w}(n, \lambda)|}{\left\langle\lambda-n^{3}\right\rangle} d \lambda\right)^{2}\right)^{1 / 2} \leq C \delta^{\theta}\|u\|_{Y_{s}}^{k+1} .
$$

Here $C$ is a constant independent of $\delta$ and $u$.

The proof of Proposition 5.2 will appear in Section 6, and is based on the idea applied by Bourgain [1993b] while proving the special case $k=2$. In the proof, we write out the detailed treatment to some subcases, and omit the similar treatment of other subcases (but it is very easy to figure out). The main 
reason we include the proof of Proposition 5.2 in Section 6 is to provide the preparation so the readers can follow the (more technical) proof of the general case $F \in C^{5}$ more easily.

We now start to derive the local well-posedness of (1-6). For this purpose, we only need to consider the well-posedness of the Cauchy problem

$$
\left\{\begin{array}{l}
u_{t}+u_{x x x}+\left(u^{k}-\int_{\mathbb{T}} u^{k} d x\right) u_{x}=0 \\
u(x, 0)=\phi(x), \quad x \in \mathbb{T}, t \in \mathbb{R} .
\end{array}\right.
$$

This is because if $v$ is a solution of (5-7), the gauge transform

$$
u(x, t):=v\left(x-\int_{0}^{t} \int_{\mathbb{T}} v^{k}(y, \tau) d y d \tau, t\right)
$$

is a solution of (1-6) with the same initial value $\phi$. Notice that this transform is invertible and preserves the initial data $\phi$. The inverse transform is

$$
v(x, t):=u\left(x+\int_{0}^{t} \int_{\mathbb{T}} u^{k}(y, \tau) d y d \tau, t\right) .
$$

It is easy to see that for any solution $u$ of (1-6), this inverse transform of $u$ defines a solution of (5-7). Hence, to establish the well-posedness of (1-6), it suffices to obtain the well-posedness of (5-7). This gauge transform was used in [Colliander et al. 2004].

By Duhamel's principle, the corresponding integral equation associated to (5-7) is

$$
u(x, t)=e^{-t \partial_{x}^{3}} \phi(x)-\int_{0}^{t} e^{-(t-\tau) \partial_{x}^{3}} w(x, \tau) d \tau,
$$

where $w$ is defined as in (5-4).

Since we are only seeking the local well-posedness, we may use a bump function to truncate the time variable. Let $\psi$ be a bump function supported in $[-2,2]$ with $\psi(t)=1,|t| \leq 1$, and let $\psi_{\delta}$ be

$$
\psi_{\delta}(t)=\psi(t / \delta)
$$

Then it suffices to find a local solution of

$$
u(x, t)=\psi_{\delta}(t) e^{-t \partial_{x}^{3}} \phi(x)-\psi_{\delta}(t) \int_{0}^{t} e^{-(t-\tau) \partial_{x}^{3}} w(x, \tau) d \tau .
$$

Let $T$ be an operator given by

$$
T u(x, t):=\psi_{\delta}(t) e^{-t \partial_{x}^{3}} \phi(x)-\psi_{\delta}(t) \int_{0}^{t} e^{-(t-\tau) \partial_{x}^{3}} w(x, \tau) d \tau .
$$

We denote the first term (the linear term) in (5-11) by $\mathscr{L} u$ and the second term (the nonlinear term) by $\mathcal{N} u$. Henceforth we represent $T u$ as $\mathscr{L} u+\mathcal{N} u$. The following two lemmas deal with $\mathscr{L} u$ and $\mathcal{N} u$ separately.

Lemma 5.3. The linear term $\mathscr{L}$ satisfies

$$
\|\mathscr{L} u\|_{Y_{s}} \leq C\|\phi\|_{H^{s}}
$$

Here $C$ is a constant independent of $\delta$. 
Lemma 5.4. The nonlinear term $\mathcal{N}$ satisfies

$$
\|\mathcal{N} u\|_{Y_{s}} \leq C\left(\|w\|_{s,-1 / 2}+\left(\sum_{n}\langle n\rangle^{2 s}\left(\int \frac{|\widehat{w}(n, \lambda)|}{\left\langle\lambda-n^{3}\right\rangle} d \lambda\right)^{2}\right)^{1 / 2}\right),
$$

where $C$ is a constant independent of $\delta$.

Lemmas 5.3 and 5.4 are considered classical and their proofs can be found in many references, such as [Colliander et al. 2004].

Proposition 5.5. Let $s>1 / 2$ and $T$ be the operator defined as in (5-11). Then there exists a positive number $\theta$ such that

$$
\|T u\|_{Y_{s}} \leq C\left(\|\phi\|_{H^{s}}+\delta^{\theta}\|u\|_{Y_{s}}^{k+1}\right) .
$$

Here $C$ is a constant independent of $\delta$.

Proof. Since $T u=\mathscr{L} u+\mathcal{N} u$, Proposition 5.5 follows from Lemmas 5.3, 5.4, and Proposition 5.2.

Proposition 5.5 yields that for $\delta$ sufficiently small, $T$ maps a ball in $Y_{S}$ into itself. Moreover, we write $\left(u^{k}-\int_{\mathbb{T}} u^{k} d x\right) u_{x}-\left(v^{k}-\int_{\mathbb{T}} v^{k} d x\right) v_{x}=\left(u^{k}-\int_{\mathbb{T}} u^{k} d x\right)(u-v)_{x}+\left(\left(u^{k}-v^{k}\right)-\int_{\mathbb{T}}\left(u^{k}-v^{k}\right) d x\right) v_{x}$ which equals

$$
\left(u^{k}-\int_{\mathbb{T}} u^{k} d x\right)(u-v)_{x}+\sum_{j=0}^{k-1}\left((u-v) u^{k-1-j} v^{j}-\int_{\mathbb{T}}(u-v) u^{k-1-j} v^{j} d x\right) v_{x} .
$$

For $k+1$ terms in (5-15), repeating similar argument as in the proof of Proposition 5.2, one obtains, for $s>1 / 2$,

$$
\|T u-T v\|_{Y_{s}} \leq C \delta^{\theta}\left(\|u\|_{Y_{S}}^{k}+\sum_{j=1}^{k-1}\|u\|_{Y_{s}}^{k-1-j}\|v\|_{Y_{S}}^{j+1}\right)\|u-v\|_{Y_{s}}
$$

Hence, for $\delta>0$ small enough, $T$ is a contraction and the local well-posedness follows from Picard's fixed-point theorem.

\section{Proof of Proposition 5.2}

Proof. From the definition of $w$ in (5-4), we may write $\hat{w}(n, \lambda)$ as

$$
\sum_{\substack{m+n_{1}+\cdots+n_{k}=n \\ n_{1}+\cdots+n_{k} \neq 0}} m \int \hat{u}\left(m, \lambda-\lambda_{1}-\cdots-\lambda_{k}\right) \hat{u}\left(n_{1}, \lambda_{1}\right) \cdots \hat{u}\left(n_{k}, \lambda_{k}\right) d \lambda_{1} \cdots d \lambda_{k} .
$$

By duality, there exists a sequence $\left\{A_{n, \lambda}\right\}$ satisfying

$$
\sum_{n \in \mathbb{Z}} \int_{\mathbb{R}}\left|A_{n, \lambda}\right|^{2} d \lambda \leq 1
$$


and $\|w\|_{s,-1 / 2}$ is bounded by

$$
\sum_{\substack{m+n_{1}+\cdots+n_{k}=n \\ n_{1}+\cdots+n_{k} \neq 0}} \int \frac{\langle n\rangle^{s}|m|}{\left\langle\lambda-n^{3}\right\rangle^{1 / 2}}\left|\hat{u}\left(m, \lambda-\lambda_{1}-\cdots-\lambda_{k}\right)\right|\left|\hat{u}\left(n_{1}, \lambda_{1}\right)\right| \cdots\left|\hat{u}\left(n_{k}, \lambda_{k}\right)\right|\left|A_{n, \lambda}\right| d \lambda_{1} \cdots d \lambda_{k} d \lambda .
$$

Since the $X_{s, b}$ is a restriction norm, we may assume that $u$ is supported in $\mathbb{T} \times[0, \delta]$. However, the inverse space-time Fourier transform $|\hat{u}|^{\vee}$ in general may not be a function with compact support. The following standard trick allows us to assume $|\hat{u}|^{\vee}$ has a compact support too. In fact, let $\eta$ be a bump function supported on $[-2 \delta, 2 \delta]$ and with $\eta(t)=1$ in $|t| \leq \delta$. Also $\hat{\eta}$ is positive. Then $u=u \eta$ and $\hat{u}=\hat{u} * \hat{\eta}$. Thus $|\hat{u}| \leq|\hat{u}| * \hat{\eta}=\left(|\hat{u}|^{\vee} \eta\right)^{\wedge}$. Whenever we need to make $|\hat{u}|^{\vee}$ supported in a small time interval, we replace $|\hat{u}|$ by $\left(|\hat{u}|^{\vee} \eta\right)^{\wedge}$ since $|\hat{u}|^{\vee} \eta$ clearly is supported on $\mathbb{\mathbb { V }} \times[-2 \delta, 2 \delta]$. This will help us gain a positive power of $\delta$ in our estimates. Moreover, without loss of generality we can assume $\left|n_{1}\right| \geq\left|n_{2}\right| \geq \cdots \geq\left|n_{k}\right|$.

The trouble occurs mainly because of the factor $|m|$ resulting from $\partial_{x} u$. The idea (inspired by Bourgain [1993b]) is that either the factor $\left\langle\lambda-n^{3}\right\rangle^{-1 / 2}$ can be used to cancel $|m|$, or $|m|$ can be distributed to some of the $\hat{u}$. More precisely, we consider three cases:

$$
\begin{aligned}
|m| & <1000 k^{2}\left|n_{2}\right|, \\
1000 k^{2}\left|n_{2}\right| & \leq|m| \leq 100 k\left|n_{1}\right|, \\
|m| & >100 k\left|n_{1}\right| .
\end{aligned}
$$

Case 1: $|m|<1000 k^{2}\left|n_{2}\right|$. This is the simplest case. In fact, in this case, it is easy to see that

$$
\langle n\rangle^{s}|m| \leq C\left\langle n_{1}\right\rangle^{s}\left\langle n_{2}\right\rangle^{1} / 2\langle m\rangle^{1} / 2
$$

Let

$$
\begin{aligned}
& F_{1}(x, t)=\sum_{n} \int \frac{\left|A_{n, \lambda}\right|}{\left\langle\lambda-n^{3}\right\rangle^{1 / 2}} e^{i \lambda t} e^{i n x} d \lambda ; \\
& G(x, t)=\sum_{n} \int\langle n\rangle^{1} / 2|\hat{u}(n, \lambda)| e^{i \lambda t} e^{i n x} d \lambda ; \\
& H(x, t)=\sum_{n} \int\langle n\rangle^{s}|\hat{u}(n, \lambda)| e^{i \lambda t} e^{i n x} d \lambda ; \\
& U(x, t)=\sum_{n} \int|\hat{u}(n, \lambda)| e^{i \lambda t} e^{i n x} d \lambda .
\end{aligned}
$$

Using (6-7), we can estimate (6-3) by

$$
C \sum_{m+n_{1}+\cdots+n_{k}=n} \int \widehat{F}(n, \lambda) \widehat{G}\left(m, \lambda-\lambda_{1}-\cdots-\lambda_{k}\right) \widehat{H}\left(n_{1}, \lambda_{1}\right) \widehat{G}\left(n_{2}, \lambda_{2}\right) \prod_{j=3}^{k} \widehat{U}\left(n_{j}, \lambda_{j}\right) d \lambda_{1} \cdots d \lambda_{k} d \lambda,
$$

which clearly equals

$$
C\left|\int_{\mathbb{T} \times \mathbb{R}} F_{1}(x, t) G(x, t)^{2} H(x, t) U(x, t)^{k-2} d x d t\right| .
$$


Apply Hölder's inequality to majorize it by

$$
C\left\|F_{1}\right\|_{4}\|G\|_{6+}^{2}\|H\|_{4}\|U\|_{6(k-2)-}^{k-2} .
$$

Since $U$ is supported on $\mathbb{T} \times[-2 \delta, 2 \delta]$, one more use of Hölder inequality yields

$$
\text { (6-3) } \leq C \delta^{\theta}\left\|F_{1}\right\|_{4}\|G\|_{6+}^{2}\|H\|_{4}\|U\|_{6(k-2)}^{k-2} .
$$

Let us recall some useful local embedding facts on $X_{s, b}$.

$$
\begin{aligned}
X_{0,1 / 3} & \subseteq L_{x, t}^{4}, & X_{0+, 1 / 2+} \subseteq L_{x, t}^{6} & (t \text { local }), \\
X_{\alpha, 1 / 2} & \subseteq L_{x, t}^{q}, & 0<\alpha<\frac{1}{2}, 2 \leq q<\frac{6}{1-2 \alpha} & (t \text { local }), \\
X_{1 / 2-\alpha, 1 / 2-\alpha} & \subseteq L_{t}^{q} L_{x}^{r}, & 0<\alpha<\frac{1}{2}, 2 \leq q, r<1 / \alpha . &
\end{aligned}
$$

The two embedding results in (6-14) are consequences of the discrete restriction estimates on $L^{4}$ and $L^{6}$, respectively (see [Bourgain 1993b] for details). (6-15) and (6-16) follow by interpolation (see [Colliander et al. 2004] for details). (6-14) yields

and

$$
\left\|F_{1}\right\|_{4} \leq C\left\|F_{1}\right\|_{0, \frac{1}{3}} \leq C\left(\sum_{n} \int\left|A_{n, \lambda}\right|^{2} d \lambda\right)^{1 / 2} \leq C,
$$

$$
\|H\|_{4} \leq C\|H\|_{0,1 / 3} \leq C\|u\|_{s, 1 / 2} \leq C\|u\|_{Y_{s}} .
$$

From (6-15) we have

$$
\|G\|_{6+} \leq C\|G\|_{0+, 1 / 2} \leq C\|u\|_{s, 1 / 2} \leq C\|u\|_{Y_{s}} .
$$

Using (6-16), we get

$$
\|U\|_{6(k-2)} \leq C\|U\|_{1 / 2-, 1 / 2-} \leq C\|u\|_{s, 1 / 2} \leq C\|u\|_{Y_{s}} .
$$

Hence, for Case 1, we have

$$
(6-3) \leq C \delta^{\theta}\|u\|_{Y_{s}}^{k+1}
$$

Case 2: $1000 k^{2}\left|n_{2}\right| \leq|m| \leq 100 k\left|n_{1}\right|$. In this case, we further consider two subcases:

$$
\begin{aligned}
& \left|m+n_{1}\right| \leq 1000 k^{2}\left|n_{2}\right|, \\
& \left|m+n_{1}\right|>1000 k^{2}\left|n_{2}\right| .
\end{aligned}
$$

If $\left|m+n_{1}\right| \leq 1000 k^{2}\left|n_{2}\right|$, we use the triangle inequality to get

$$
|n|=\left|m+n_{1}+n_{2}+\cdots+n_{k}\right| \leq C\left|n_{2}\right| \text {. }
$$

Hence we have

$$
\langle n\rangle^{s}|m| \leq C\left\langle n_{2}\right\rangle^{s}\langle m\rangle^{1 / 2}\left\langle n_{1}\right\rangle^{1 / 2}
$$

Thus this subcase can be treated exactly the same as Case 1 . We omit the details. 
In the second subcase, $\left|m+n_{1}\right|>1000 k^{2}\left|n_{2}\right|$, the crucial arithmetic observation is

$$
n^{3}-\left(m^{3}+n_{1}^{3}+\cdots+n_{k}^{3}\right)=3\left(m+n_{1}\right)(m+a)\left(n_{1}+a\right)+a^{3}-\left(n_{2}^{3}+\cdots+n_{k}^{3}\right),
$$

where $a=n_{2}+\cdots+n_{k}$. This observation can be easily verified since $n=m+n_{1}+\cdots+n_{k}$. From (6-5) and (6-19), we get

$$
\left|n^{3}-\left(m^{3}+n_{1}^{3}+\cdots+n_{k}^{3}\right)\right| \geq C k^{2}\left\langle n_{2}\right\rangle|m|\left|n_{1}\right| \geq C k|m|^{2} .
$$

This implies that at least one of following statements holds:

$$
\begin{aligned}
\left|\lambda-n^{3}\right| & \geq C|m|^{2}, \\
\left|\left(\lambda-\lambda_{1}-\cdots-\lambda_{k}\right)-m^{3}\right| & \geq C|m|^{2},
\end{aligned}
$$

there exists an $i \in\{1, \ldots, k\}$ such that $\left|\lambda_{i}-n_{i}^{3}\right| \geq C|m|^{2}$.

For (6-24), (6-3) can be bounded by

$$
\sum_{m+n_{1}+\cdots+n_{k}=n} \int\left\langle n_{1}\right\rangle^{s}\left|\hat{u}\left(m, \lambda-\lambda_{1}-\cdots-\lambda_{k}\right)\right|\left|\hat{u}\left(n_{1}, \lambda_{1}\right)\right| \cdots\left|\hat{u}\left(n_{k}, \lambda_{k}\right)\right|\left|A_{n, \lambda}\right| d \lambda_{1} \cdots d \lambda_{k} d \lambda .
$$

Let $F_{2}$ be defined by

$$
F_{2}(x, t)=\sum_{n} \int\left|A_{n, \lambda}\right| e^{i \lambda t} e^{i n x} d \lambda
$$

Then we represent (6-27) as

$$
\sum_{m+n_{1}+\cdots+n_{k}=n} \int \widehat{F}_{2}(n, \lambda) \widehat{U}\left(m, \lambda-\lambda_{1}-\cdots-\lambda_{k}\right) \widehat{H}\left(n_{1}, \lambda_{1}\right) \prod_{j=2}^{k} \widehat{U}\left(n_{j}, \lambda_{j}\right) d \lambda_{1} \cdots d \lambda_{k} d \lambda .
$$

Here $H$ and $U$ are the functions defined in (6-10) and (6-11). Clearly (6-29) equals

$$
\int_{\mathbb{T} \times \mathbb{R}} F_{2}(x, t) H(x, t) U(x, t)^{k} d x d t .
$$

Utilizing Hölder's inequality, we estimate it further by

$$
\left\|F_{2}\right\|_{2}\|H\|_{4}\|U\|_{4 k}^{k} \leq C \delta^{\theta}\|u\|_{Y_{s}}^{k+1} .
$$

This yields the desired estimate for subcase (6-24).

One can similarly complete the proofs of subcases (6-25) and (6-26), and hence the proof of Case 2. Case 3: $|m|>100 k\left|n_{1}\right|$. The arithmetic observation (6-22) again plays an important role. In this case, let us further consider two subcases:

$$
\begin{aligned}
& |m|^{2} \leq 1000 k^{2}\left|n_{2}\right|^{2}\left|n_{3}\right|, \\
& |m|^{2}>1000 k^{2}\left|n_{2}\right|^{2}\left|n_{3}\right| .
\end{aligned}
$$

For the first subcase, we observe that, from (6-32), 


$$
|m|^{2} \leq C\left|n_{1}\right|\left|n_{2}\right|\left|n_{3}\right|
$$

since $\left|n_{2}\right| \leq\left|n_{1}\right|$. Hence we have

$$
|m|=|m|^{1 / 2}|m|^{2 / 3} \leq C|m|^{1 / 3}\left|n_{1}\right|^{1 / 3}\left|n_{2}\right|^{1 / 3}\left|n_{3}\right|^{1 / 3} .
$$

This immediately implies

$$
\langle n\rangle^{s}|m| \leq C|m|^{s+1} \leq\langle m\rangle^{(s+1) / 3}\left\langle n_{1}\right\rangle^{(s+1) / 3}\left\langle n_{2}\right\rangle^{(s+1) / 3}\left\langle n_{3}\right\rangle^{(s+1) / 3} .
$$

Note that $(s+1) / 3<s$ for $s>1 / 2$. By distributing the four factors to the corresponding functions, one can mimic the proof of Case 1 to finish subcase (6-32).

We now turn to the contribution of (6-33). Clearly we have

$$
\left|\left(n_{2}+\cdots+n_{k}\right)^{3}-\left(n_{2}^{3}+\cdots+n_{k}^{3}\right)\right| \leq 10 k\left|n_{2}\right|^{2}\left|n_{3}\right|,
$$

since $\left|n_{2}\right| \geq\left|n_{3}\right| \geq \cdots \geq\left|n_{k}\right|$. From the crucial arithmetic observation (6-22), (6-36), and (6-33), we have

$$
\left|n^{3}-\left(m^{3}+n_{1}^{3}+\cdots+n_{k}^{3}\right)\right| \geq C k|m|^{2}
$$

This is the same as (6-23). Hence we again reduce the problems to (6-24), (6-25), and (6-26), which were all done in Case 2. Therefore Case 3 is finished.

Putting all the cases together, we obtain

$$
\|w\|_{s,-1 / 2} \leq C \delta^{\theta}\|u\|_{Y_{s}}^{k+1}
$$

Finally we need to estimate

$$
\left(\sum_{n}\langle n\rangle^{2 s}\left(\int \frac{|\widehat{w}(n, \lambda)|}{\left\langle\lambda-n^{3}\right\rangle} d \lambda\right)^{2}\right)^{1 / 2}
$$

Let $\left\{A_{n}\right\}$ be a sequence with

$$
\left(\sum_{n}\left|A_{n}\right|^{2}\right)^{1 / 2} \leq 1
$$

By duality, it suffices to estimate

$$
\sum_{\substack{m+n_{1}+\cdots+n_{k}=n \\ n_{1}+\cdots+n_{k} \neq 0}} \int \frac{\langle n\rangle^{s}|m|}{\left\langle\lambda-n^{3}\right\rangle}\left|\hat{u}\left(m, \lambda-\lambda_{1}-\cdots-\lambda_{k}\right)\right|\left|\hat{u}\left(n_{1}, \lambda_{1}\right)\right| \cdots\left|\hat{u}\left(n_{k}, \lambda_{k}\right)\right|\left|A_{n}\right| d \lambda_{1} \cdots d \lambda_{k} d \lambda .
$$

By the same idea and similar techniques, one can bound (6-40) by mimicking the treatment of (6-3) and get

$$
\left(\sum_{n}\langle n\rangle^{2 s}\left(\int \frac{|\widehat{w}(n, \lambda)|}{\left\langle\lambda-n^{3}\right\rangle} d \lambda\right)^{2}\right)^{1 / 2} \leq C \delta^{\theta}\|u\|_{Y_{s}}^{k+1}
$$

We complete the proof of Proposition 5.2 by combining (6-38) and (6-41). 


\section{Proof of Theorem $\mathbf{1 . 3}$}

The argument is similar to that in Section 5. By using a gauge transform as in (5-8) with $v^{k}$ replaced by $F(v)$, the well-posedness of (1-7) is equivalent to the well-posedness of the following equation:

$$
\left\{\begin{array}{l}
u_{t}+u_{x x x}+\left(F(u)-\int_{\mathbb{T}} F(u) d x\right) u_{x}=0, \\
u(x, 0)=\phi(x), \quad x \in \mathbb{T}, t \in \mathbb{R} .
\end{array}\right.
$$

Now the nonlinear function $w$ is defined by

$$
w=\partial_{x} u\left(F(u)-\int_{\mathbb{T}} F(u) d x\right) .
$$

Let $T_{F}$ be an operator given by

$$
T_{F} u(x, t):=\psi_{\delta}(t) e^{-t \partial_{x}^{3}} \phi(x)-\psi_{\delta}(t) \int_{0}^{t} e^{-(t-\tau) \partial_{x}^{3}} w(x, \tau) d \tau .
$$

As in Section 5, the local well-posedness is a consequence of the following proposition.

Proposition 7.1. Let $s>1 / 2$. There exists $\theta>0$ such that, for the nonlinear function $w$ given by (7-2) and any $u$ satisfying $\|u\|_{Y_{s}} \leq C_{0}\|\phi\|_{H^{s}}$,

$$
\|w\|_{s,-1 / 2}+\left(\sum_{n}\langle n\rangle^{2 s}\left(\int \frac{|\widehat{w}(n, \lambda)|}{\left\langle\lambda-n^{3}\right\rangle} d \lambda\right)^{2}\right)^{1 / 2} \leq C\left(\|\phi\|_{H^{s}}, F\right) \delta^{\theta}\|u\|_{Y_{s}}^{4},
$$

provided $F \in C^{5}$. Here $C_{0}$ is a suitably large constant, and $C\left(\|\phi\|_{H^{s}}, F\right)$ is a constant independent of $\delta$ and $u$, but which may depend on $\|\phi\|_{H^{s}}$ and $F$.

The constant $C\left(\|\phi\|_{H^{s}}, F\right)$ will be specified in the proof of Proposition 7.1, which we postpone to Section 8. We now return to the proof of Theorem 1.3. Proposition 7.1 implies that for $\delta$ sufficiently small, $T$ maps a ball

$$
\left\{u \in Y_{s}:\|u\|_{Y_{s}} \leq C_{0}\|\phi\|_{H^{s}}\right\}
$$

into itself. Moreover, using Lemma 5.4 and repeating similar argument as in the proof of Proposition 7.1, one obtains, for $s>1 / 2$ and $F \in C^{5}$,

$$
\left\|T_{F} u-T_{F} v\right\|_{Y_{s}} \leq \delta^{\theta} C\left(\|\phi\|_{H^{s}}, F\right)\|u-v\|_{Y_{s}}
$$

for all $u, v$ in the ball $\left\{u \in Y_{s}:\|u\|_{Y_{s}} \leq C_{0}\|\phi\|_{H^{s}}\right\}$. Therefore, for $\delta>0$ small enough, $T_{F}$ is a contraction on the ball and the local well-posedness again follows from Picard's fixed-point theorem. This completes the proof of Theorem 1.3.

\section{Proof of Proposition 7.1}

First we introduce a decomposition of $F(u)$ which was used by Bourgain. Let $K$ be a dyadic number, and define a Fourier multiplier operator $P_{K}$ by setting

$$
P_{K} u(x, t)=\int \psi_{K}(y) u(x-y, t) d y .
$$


Here the Fourier transform of $\psi_{K}$ is a standard bump function supported on $[-2 K, 2 K]$ and $\widehat{\psi_{K}}(x)=1$ for $x \in[-K, K]$. Let $u_{K}$ denote the Littlewood-Paley Fourier multiplier, that is,

$$
u_{K}=P_{K} u-P_{K / 2} u \text {. }
$$

Then we may decompose $F(u)$ by

$$
F(u)=\sum_{K}\left(F\left(P_{K} u\right)-F\left(P_{K / 2} u\right)\right)=\sum_{K} F_{1}\left(P_{K} u, P_{K / 2} u\right) u_{K}+R_{1},
$$

where $R_{1}$ is a function independent of the space variable $x$. Repeating this procedure for $F_{1}$, we obtain

$$
\begin{aligned}
F(u) & =\sum_{K_{1} \geq K_{2}} F_{2}\left(P_{2 K_{2}} u, \ldots, P_{K_{2} / 4} u\right) u_{K_{1}} u_{K_{2}}+\sum_{K_{1}} R_{2} u_{K_{1}}+R_{1} \\
& =\sum_{K_{1} \geq K_{2} \geq K_{3}} F_{3}\left(P_{4 K_{3}} u, \ldots, P_{K_{3} / 8} u\right) u_{K_{1}} u_{K_{2}} u_{K_{3}}+\sum_{K_{1} \geq K_{2}} R_{3} u_{K_{1}} u_{K_{2}}+\sum_{K_{1}} R_{2} u_{K_{1}}+R_{1}
\end{aligned}
$$

where $R_{1}, R_{2}, R_{3}$ are functions independent of the space variable. Set

$$
G_{K_{3}}(x, t)=F_{3}\left(P_{4 K_{3}} u, \ldots, P_{K_{3} / 8} u\right) .
$$

Hence we represent $w$, defined in (7-2), as

$$
\begin{aligned}
w= & \sum_{K_{0}, K_{1} \geq K_{2} \geq K_{3}} \partial_{x} u_{K_{0}}\left(u_{K_{1}} u_{K_{2}} u_{K_{3}} G_{K_{3}}-\int_{\mathbb{T}} u_{K_{1}} u_{K_{2}} u_{K_{3}} G_{K_{3}} d x\right) \\
& +\sum_{K_{0}, K_{1} \geq K_{2}} \partial_{x} u_{K_{0}}\left(u_{K_{1}} u_{K_{2}}-\int_{\mathbb{T}} u_{K_{1}} u_{K_{2}} d x\right) R_{3}+\sum_{K_{0}, K_{1}} \partial_{x} u_{K_{0}}\left(u_{K_{1}}-\int_{\mathbb{T}} u_{K_{1}} d x\right) R_{2} .
\end{aligned}
$$

The main contribution of $w$ is from the first term. The remaining terms can be handled by the method presented in Section 6, because $R_{2}, R_{3}$ are functions independent of the space variable $x$ (actually they only depend on the conserved quantity $\int_{\mathbb{T}} u d x$ ). Hence in what follows we only focus on estimating the first term - the most difficult one. Denote the first term by $w_{1}$ :

$$
w_{1}=\sum_{K_{0}, K_{1} \geq K_{2} \geq K_{3}} \partial_{x} u_{K_{0}}\left(u_{K_{1}} u_{K_{2}} u_{K_{3}} G_{K_{3}}-\int_{\mathbb{T}} u_{K_{1}} u_{K_{2}} u_{K_{3}} G_{K_{3}} d x\right) .
$$

We should prove

$$
\left\|w_{1}\right\|_{s,-1 / 2}+\left(\sum_{n}\langle n\rangle^{2 s}\left(\int \frac{\left|\hat{w}_{1}(n, \lambda)\right|}{\left\langle\lambda-n^{3}\right\rangle} d \lambda\right)^{2}\right)^{1 / 2} \leq C \delta^{\theta} C\left(\|\phi\|_{H^{s}}, F\right)\|u\|_{Y_{s}}^{4} .
$$

In order to specify the constant $C\left(\|\phi\|_{H^{s}}, F\right)$, we define $\mathfrak{M}$ by setting

$$
\mathfrak{M}=\sup \left\{\left|D^{\alpha} F_{3}\left(u_{1}, \ldots, u_{6}\right)\right|: u_{j} \text { satisfies }\left\|u_{j}\right\|_{Y_{s}} \leq C_{0}\|\phi\|_{H^{s}} \text { for all } j=1, \ldots, 6 ; \alpha\right\} .
$$

Here $D^{\alpha}=\partial_{x_{1}}^{\alpha_{1}} \ldots \partial_{x_{6}}^{\alpha_{6}}$ and $\alpha$ is taken over all tuples $\left(\alpha_{1}, \ldots, \alpha_{6}\right) \in(\mathbb{N} \cup\{0\})^{6}$ with $\sum\left|\alpha_{j}\right| \leq 2 . \mathfrak{M}$ is a real number. This is because, for $s>1 / 2,\|u\|_{Y_{s}} \leq C_{0}\|\phi\|_{H^{s}}$ yields that $u$ is bounded by $C\|\phi\|_{H^{s}}$, and the previous claim follows from $F_{3} \in C^{2}$. 
In order to bound $\left\|w_{1}\right\|_{s,-1 / 2}$, by duality, it suffices to bound

$$
\begin{aligned}
& \sum_{\substack{K_{0}, K_{1} \geq K_{2} \geq K_{3} \\
n_{0}+n_{1}+n_{2}+n_{3}+m=n \\
n_{1}+n_{2}+n_{3}+m \neq 0}} \int \frac{A_{n, \lambda}\langle n\rangle^{s} n_{0}}{\left\langle\lambda-n^{3}\right\rangle^{1 / 2}} \hat{u}_{K_{0}}\left(n_{0}, \lambda-\lambda_{1}-\lambda_{2}-\lambda_{3}-\mu\right) \\
& \cdot \prod_{j=1}^{3} \hat{u}_{K_{j}}\left(n_{j}, \lambda_{j}\right) \widehat{G_{K_{3}}}(m, \mu) d \lambda_{1} \cdots d \lambda_{4} d \lambda d \mu,
\end{aligned}
$$

where $A_{n, \lambda}$ satisfies

$$
\sum_{n} \int\left|A_{n, \lambda}\right|^{2} d \lambda=1
$$

The trouble maker is $G_{K_{3}}$ since there is no way to find a suitable upper bound for its $X_{s, b}$ norm. Because of this, the method in Section 6 is no longer valid, and we have to treat $m$ and $\mu$ differently from $n$ and $\lambda$, respectively. A delicate analysis must be done to overcome the difficulty caused by $G_{K_{3}}$. For simplicity, we assume that $\delta=1$. One can modify the argument to gain a decay of $\delta^{\theta}$ by using the technical treatment from Section 6.

For a dyadic number $M$, define the Littlewood-Paley Fourier multiplier by

$$
g_{K_{3}, M}=P_{M} G_{K_{3}}-P_{M / 2} G_{K_{3}}=\left(G_{K_{3}}\right)_{M} .
$$

Let $v$ be defined by

$$
v(x, t)=\sum_{n} \int \frac{A_{n, \lambda}}{\left\langle\lambda-n^{3}\right\rangle^{1 / 2}} e^{i \lambda t} e^{i n x} d \lambda .
$$

To estimate (8-7), it suffices to estimate

$$
\begin{aligned}
\sum_{\substack{K, K_{0}, K_{1} \geq K_{2} \geq K_{3}, M \\
n_{0}+n_{1}+n_{2}+n_{3}+m=n \\
n_{1}+n_{2}+n_{3}+m \neq 0}} \int \widehat{\left\langle\partial_{x}\right\rangle^{s} v_{K}}(n, \lambda) \widehat{\partial_{x} u_{K_{0}}}\left(n_{0}, \lambda-\lambda_{1}-\lambda_{2}-\lambda_{3}-\mu\right) \\
\cdot \prod_{j=1}^{3} \hat{u}_{K_{j}}\left(n_{j}, \lambda_{j}\right) \widehat{g_{K_{3}, M}}(m, \mu) d \lambda_{1} \cdots d \lambda_{4} d \lambda d \mu .
\end{aligned}
$$

Here $K$ is a dyadic number.

As we did in Section 6, we consider three cases:

$$
\begin{aligned}
K_{0} & <2^{100} K_{2} \\
2^{100} K_{2} & \leq K_{0} \leq 2^{10} K_{1} \\
K_{0} & >2^{10} K_{1} .
\end{aligned}
$$

The rest of the paper is devoted to a proof of these three cases. In what follows, we will only provide the details for the estimates of $\left\|w_{1}\right\|_{s,-1 / 2}$ with $1 / 2<s<1$ (the case $s \geq 1$ is easier). For the desired estimate of

$$
\left(\sum_{n}\langle n\rangle^{2 s}\left(\int \frac{\left|\hat{w}_{1}(n, \lambda)\right|}{\left\langle\lambda-n^{3}\right\rangle} d \lambda\right)^{2}\right)^{1 / 2},
$$


simply replace $v$ by

$$
v_{1}(x, t)=\sum_{n} \int \frac{C_{n, \lambda} A_{n}}{\left\langle\lambda-n^{3}\right\rangle} e^{i \lambda t} e^{i n x} d \lambda,
$$

and then the desired estimate follows similarly. Here $C_{n, \lambda} \in \mathbb{C}$ satisfies $\sup _{\lambda}\left|C_{n}, \lambda\right| \leq 1$ and $\left\{A_{n}\right\}$ satisfies $\sum_{n}\left|A_{n}\right|^{2} \leq 1$.

\section{Proof of case (8-11)}

In this case, we should consider further two subcases:

$$
\begin{aligned}
& M \leq 2^{10} K_{1}, \\
& M>2^{10} K_{1} .
\end{aligned}
$$

For the contribution of (9-1), noticing that $K \leq C K_{1}$ in this subcase, we estimate (8-10) by

$$
\sum_{K_{1} \geq K_{2} \geq K_{3}} \int_{\mathbb{T} \times \mathbb{R}}\left|\left(\sum_{K \leq C K_{1}} \partial_{x}^{s} v_{K}\right)\left(\sum_{K_{0} \leq C K_{2}} \partial_{x} u_{K_{0}}\right) u_{K_{1}} u_{K_{2}} u_{K_{3}}\left(P_{2^{10} K_{1}} G_{K_{3}}\right)\right| d x d t,
$$

which is bounded by

$$
\sum_{K_{3}}\left\|u_{K_{3}}\right\|_{\infty}\left\|G_{K_{3}}\right\|_{\infty} \int_{\mathbb{T} \times \mathbb{R}} \sum_{K_{1}} \sum_{K \leq C K_{1}} K^{s} v_{K}^{*}\left|u_{K_{1}}\right| \sum_{K_{2}} \sum_{K_{0} \leq C K_{2}} K_{0} u_{K_{0}}^{*}\left|u_{K_{2}}\right| d x d t,
$$

where $f^{*}$ stands for the Hardy-Littlewood maximal function of $f$. By the Schur's test, (9-4) can be estimated by

$$
\begin{aligned}
\sum_{K_{3}} K_{3}^{-(2 s-1) / 2}\|u\|_{Y_{s}} \mathfrak{M} \int\left(\sum_{K}\left|v_{K}^{*}\right|^{2}\right)^{1 / 2}\left(\sum_{K_{1}} K_{1}^{2 s}\left|u_{K_{1}}\right|^{2}\right)^{1 / 2} & \cdot\left(\sum_{K_{0}} K_{0}\left|u_{K_{0}}^{*}\right|^{2}\right)^{1 / 2}\left(\sum_{K_{2}} K_{2}\left|u_{K_{2}}\right|^{2}\right)^{1 / 2} d x d t .
\end{aligned}
$$

Since $s>1 / 2$, we obtain, by a use of Hölder's inequality, that (9-4) is majorized by

$$
C \mathfrak{M}\|u\|_{Y_{s}}\left\|\left(\sum_{K}\left|v_{K}^{*}\right|^{2}\right)^{1 / 2}\right\|\left\|\left(\sum_{K_{1}} K_{1}^{2 s}\left|u_{K_{1}}\right|^{2}\right)^{1 / 2}\right\|_{4}\left\|\left(\sum_{K_{0}} K_{0}\left|u_{K_{0}}^{*}\right|^{2}\right)^{1 / 2}\right\|\left\|\left(\sum_{K_{2}} K_{2}\left|u_{K_{2}}\right|^{2}\right)^{1 / 2}\right\|_{4} \text {. }
$$

Observe that

$$
\left\|\left(\sum_{K}\left|v_{K}^{*}\right|^{2}\right)^{1 / 2}\right\|_{4} \leq\left\|\left(\sum_{K}\left|v_{K}\right|^{2}\right)^{1 / 2}\right\|_{4} \leq C\|v\|_{4} \leq C\|v\|_{0,1 / 3} \leq C .
$$

Here the first inequality is obtained by using Fefferman and Stein's vector-valued inequality on the maximal function, and the second is a consequence of the classical Littlewood-Paley theorem. Similarly,

$$
\left\|\left(\sum_{K_{0}} K_{0}\left|u_{K_{0}}^{*}\right|^{2}\right)^{1 / 2}\right\|_{4} \leq\left\|\left(\sum_{K_{0}} K_{0}\left|u_{K_{0}}\right|^{2}\right)^{1 / 2}\right\|_{4} \leq C\left\|\partial_{x}^{1 / 2} u\right\|_{4} \leq C\|u\|_{1 / 2,1 / 3} \leq C\|u\|_{Y_{s}}
$$


and

$$
\left\|\left(\sum_{K_{1}} K_{1}^{2 s}\left|u_{K_{1}}\right|^{2}\right)^{1 / 2}\right\|_{4} \leq C\left\|\partial_{x}^{s} u\right\|_{4} \leq C\|u\|_{s, 1 / 3} \leq C\|u\|_{Y_{s}} .
$$

Hence, from (9-7), (9-8) and (9-9), we have

$$
(8-10) \leq C \mathfrak{M}\|u\|_{Y_{s}}^{4} .
$$

For the contribution of (9-2), since in this subcase $K \leq C M$, we estimate (8-10) by

$$
\sum_{K_{1}}\left\|u_{K_{1}}\right\|_{\infty} \int_{\mathbb{T} \times \mathbb{R}} \sum_{K_{3} \leq K_{1}}\left|u_{K_{3}}\right| \sum_{M} \sum_{K \leq C M} K^{s} v_{K}^{*}\left|g_{K_{3}, M}\right| \sum_{K_{2}} \sum_{K_{0} \leq C K_{2}} K_{0} u_{K_{0}}^{*}\left|u_{K_{2}}\right| d x d t
$$

which is bounded by

$$
\begin{aligned}
& \sum_{K_{1}} K_{1}^{-(2 s-1) / 2}\|u\|_{Y_{s}} \int_{\mathbb{T} \times \mathbb{R}} \sum_{K_{3} \leq K_{1}}\left|u_{K_{3}}\right|\left(\sum_{K}\left|v_{K}^{*}\right|^{2}\right)^{1 / 2}\left(\sum_{M} M^{2 s}\left|g_{K_{3}, M}\right|^{2}\right)^{1 / 2} \\
& \cdot\left(\sum_{K_{0}} K_{0}\left|u_{K_{0}}^{*}\right|^{2}\right)^{1 / 2}\left(\sum_{K_{2}} K_{2}\left|u_{K_{2}}\right|^{2}\right)^{1 / 2} d x d t .
\end{aligned}
$$

By a use of the Cauchy-Schwarz inequality, (9-12) is estimated by

$$
\begin{aligned}
& \sum_{K_{1}} K_{1}^{-(2 s-1) / 2}\|u\|_{Y_{s}} \int_{\mathbb{T} \times \mathbb{R}}\left(\sum_{K}\left|v_{K}^{*}\right|^{2}\right)^{1 / 2}\left(\sum_{K_{0}} K_{0}\left|u_{K_{0}}^{*}\right|^{2}\right)^{1 / 2}\left(\sum_{K_{2}} K_{2}\left|u_{K_{2}}\right|^{2}\right)^{1 / 2} \\
& \cdot\left(\sum_{K_{3}} K_{3}^{2 s}\left|u_{K_{3}}\right|^{2}\right)^{1 / 2}\left(\sum_{K_{3} \leq K_{1}} \sum_{M} \frac{M^{2 s}}{K_{3}^{2 s}}\left|g_{K_{3}, M}\right|^{2}\right)^{1 / 2} d x d t .
\end{aligned}
$$

Using Hölder's inequality, we then bound it further by

$$
\begin{aligned}
\sum_{K_{1}} K_{1}^{-(2 s-1) / 2}\|u\|_{Y_{s}} \|\left(\sum_{K}\left|v_{K}^{*}\right|^{2}\right)^{1 / 2} & \|\|_{4}\left\|\left(\sum_{K_{2}} K_{0}\left|u_{K_{0}}^{*}\right|^{2}\right)^{1 / 2}\right\|_{6}\left\|\left(\sum_{K_{2}} K_{2}\left|u_{K_{2}}\right|^{2}\right)^{1 / 2}\right\|_{6} \\
\cdot & \left\|\left(\sum_{K_{3}} K_{3}^{2 s}\left|u_{K_{3}}\right|^{2}\right)^{1 / 2}\right\|_{4}\left\|\left(\sum_{K_{3} \leq K_{1}} \sum_{M} \frac{M^{2 s}}{K_{3}^{2 s}}\left|g_{K_{3}, M}\right|^{2}\right)^{1 / 2}\right\|_{6},
\end{aligned}
$$

which is majorized by

$$
\sum_{K_{1}} K_{1}^{-(2 s-1) / 2}\|u\|_{Y_{S}}^{4} \sum_{K_{3} \leq K_{1}} K_{3}^{-s}\left\|\left(\sum_{M} M^{2 s}\left|g_{K_{3}, M}\right|^{2}\right)^{1 / 2}\right\|_{6} \leq \sum_{K_{1}} K_{1}^{-(2 s-1) / 2}\|u\|_{Y_{s}}^{4} \sum_{K_{3} \leq K_{1}} K_{3}^{-s}\left\|\partial_{x}^{s} G_{K_{3}}\right\|_{\infty} .
$$

From the definition of $G_{K_{3}}$, we have

$$
\partial_{x} G_{K_{3}}(x, t)=\mathrm{O}\left(\mathfrak{M} K_{3}\right)\|u\|_{Y_{s}}=\mathrm{O}\left(\mathfrak{M} K_{3}\right)\|\phi\|_{H^{s}} .
$$

Hence, for $s<1$,

$$
\left\|\partial_{x}^{s} G_{K_{3}}\right\|_{\infty} \leq C \mathfrak{M} K_{3}^{s}\|\phi\|_{H^{s}}
$$


Since $s>1 / 2$, we then have

$$
(9-14) \leq C \mathfrak{M}\|\phi\|_{H^{s}} \sum_{K_{1}} K_{1}^{-(2 s-1) / 2+\varepsilon}\|u\|_{Y_{s}}^{4} \leq C \mathfrak{M}\|\phi\|_{H^{s}}\|u\|_{Y_{s}}^{4} .
$$

This completes our discussion of Case (8-11).

\section{Proof of case $(8-12)$}

In this case, it suffices to consider the following subcases:

$$
\begin{aligned}
& K \leq 2^{10} K_{2}, \\
& K \leq 2^{10} M, \\
& K>2^{9}\left(K_{2}+M\right) \quad \text { and } \quad K_{3} \geq K_{0}^{1 / 2}, \\
& K>2^{9}\left(K_{2}+M\right), \quad K_{3} \leq K_{0}^{1 / 2}, \quad \text { and } \quad M \geq 2^{-10} K_{0}^{2 / 3}, \\
& K>2^{9}\left(K_{2}+M\right), \quad K_{3} \leq K_{0}^{1 / 2}, \quad \text { and } \quad M<2^{-10} K_{0}^{2 / 3} .
\end{aligned}
$$

The first two cases can be handled in exactly the same way as cases (9-1) and (9-2).

For case (10-3), observe that (8-12) and (10-3) imply

$$
K \leq C K_{1}
$$

and

$$
K_{0}^{1 / 2} \leq K_{2}^{1 / 2} K_{3}^{1 / 2}
$$

Hence (8-10) is bounded by

$$
\int \sum_{K_{1}} \sum_{K \leq C K_{1}} K^{s} v_{K}^{*}\left|u_{K_{1}}\right| \sum_{\substack{K_{0} \geq K_{2} \geq K_{3} \\ K_{0} \leq K_{3}^{2}}} K_{0} u_{K_{0}}^{*}\left|u_{K_{2}}\right|\left|u_{K_{3}}\right|\left\|G_{K_{3}}\right\|_{\infty} d x d t
$$

Applying Hölder's inequality, we estimate (10-8) by

$$
C \mathfrak{M} \int\left(\sum_{K}\left|v_{K}^{*}\right|^{2}\right)^{1 / 2}\left(\sum_{K_{1}} K_{1}^{2 s}\left|u_{K_{1}}\right|^{2}\right)^{1 / 2} \prod_{j=0,2,3}\left(\sum_{K_{j}} K_{j}^{1+\varepsilon}\left|u_{K_{j}}\right|^{2}\right)^{1 / 2} d x d t
$$

One more use of Hölder's inequality yields that (10-8) is bounded by

$$
C \mathfrak{M}\left\|\left(\sum_{K}\left|v_{K}\right|^{2}\right)^{1 / 2}\right\|_{4}\left\|\left(\sum_{K_{1}} K_{1}^{2 s}\left|u_{K_{1}}\right|^{2}\right)^{1 / 2}\right\|_{4} \prod_{j=0,2,3}\left\|\left(\sum_{K_{j}} K_{j}^{1+\varepsilon}\left|u_{K_{j}}\right|^{2}\right)^{1 / 2}\right\|_{6} .
$$

Hence we obtain

$$
(10-8) \leq C \mathfrak{M}\|u\|_{Y_{s}}^{4}
$$

This finishes the proof of (10-3). 
For case (10-4), we estimate (8-10) by

$$
\sum_{K_{2}, K_{3}} \int \sum_{K_{1}} \sum_{K \leq C K_{1}} K^{s} v_{K}^{*}\left|u_{K_{1}}\right| \sum_{K_{0}} K_{0}\left|u_{K_{0}}^{*}\right|\left|u_{K_{2}}\right|\left|u_{K_{3}}\right| \sum_{M \geq C K_{0}^{2 / 3}}\left|g_{K_{3}, M}\right| d x d t
$$

which is dominated by

$$
\begin{aligned}
C \sum_{K_{2}, K_{3}} \int\left(\sum_{K}\left|v_{K}^{*}\right|^{2}\right)^{1 / 2}\left(\sum_{K_{1}} K_{1}^{2 s}\left|u_{K_{1}}\right|^{2}\right)^{1 / 2} & \left|u_{K_{2}}\right|\left|u_{K_{3}}\right| \\
& \cdot\left(\sum_{K_{0}} K_{0}\left|u_{K_{0}}^{*}\right|^{2}\right)^{1 / 2}\left(\sum_{M} M^{3 / 2}\left|g_{K_{3}, M}\right|^{2}\right)^{1 / 2} d x d t .
\end{aligned}
$$

By Hölder's inequality with $L^{4}$ norms for the first two functions in the integrand, $L^{6+}$ norms for the next three functions, and an $L^{p}$ norm (very large $p$ ) for the last one, (10-12) is dominated by

$$
C\|u\|_{Y_{s}} \sum_{K_{2}, K_{3}}\left\|u_{K_{2}}\right\|_{6+}\left\|u_{K_{3}}\right\|_{6+}\left\|\left(\sum_{K_{0}} K_{0}\left|u_{K_{0}}^{*}\right|^{2}\right)^{1 / 2}\right\|_{6+}\left\|\partial_{x}^{3 / 4} G_{K_{3}}\right\|_{\infty} .
$$

Applying (9-16), we estimate (10-12) by

$C \mathfrak{M}\|\phi\|_{H_{s}}\|u\|_{Y_{s}}^{2} \prod_{j=2}^{3} \sum_{K_{j}} K_{j}^{3 / 8}\left\|u_{K_{j}}\right\|_{6+} \leq C \mathfrak{M}\|\phi\|_{H_{s}}\|u\|_{Y_{s}}^{2} \prod_{j=2}^{3} \sum_{K_{j}} K_{j}^{3 / 8}\left\|u_{K_{j}}\right\|_{0+, 1 / 2} \leq C \mathfrak{M}\|\phi\|_{H_{s}}\|u\|_{Y_{s}}^{4}$,

as desired. This completes the discussion of (10-4).

We now turn to case (10-5). In this case, we have

$$
\left|n_{0}+n_{1}\right|+2 K_{2}+M \geq|n| \geq K / 2 \geq 2^{8}\left(K_{2}+M\right),
$$

which implies

$$
\left|n_{0}+n_{1}\right| \geq 2^{5}\left(K_{2}+M\right) .
$$

Notice that

$$
\begin{aligned}
& \left(n_{0}+n_{1}+n_{2}+n_{3}+m\right)^{3}-n_{0}^{3}-n_{1}^{3}-n_{2}^{3}-n_{3}^{3}-m^{3} \\
& \quad=3\left(n_{0}+n_{1}\right)\left(n_{0}+n_{2}+n_{3}+m\right)\left(n_{1}+n_{2}+n_{3}+m\right)+\left(n_{2}+n_{3}+m\right)^{3}-n_{2}^{3}-n_{3}^{3}-m^{3} .
\end{aligned}
$$

From (10-15), (10-16), and (10-5), we obtain

$$
\left|n^{3}-n_{0}^{3}-n_{1}^{3}-n_{2}^{3}-n_{3}^{3}-m^{3}\right| \geq C\left(K_{2}+M\right) K_{0} K_{1} \geq C K_{0} K_{1} \geq C K_{0}^{2} .
$$

Hence one of the following four statements must be true:

$$
\begin{aligned}
\left|\lambda-n^{3}\right| & \geq K_{0}^{2}, \\
\left|\left(\lambda-\lambda_{1}-\lambda_{2}-\lambda_{3}-\mu\right)-n_{0}^{3}\right| & \geq K_{0}^{2},
\end{aligned}
$$

there exists an $i \in\{1,2,3\}$ such that $\left|\lambda_{i}-n_{i}^{3}\right| \geq K_{0}^{2}$,

$$
|\mu| \geq K_{0}^{2} \text {. }
$$


For case (10-18), we set

$$
\tilde{v}(x, t)=\left(\hat{v} \mathbf{1}_{\left|\lambda-n^{3}\right| \geq K_{0}^{2}}\right)^{\vee}(x, t) .
$$

We then estimate (8-10) by

$$
\sum_{K_{2}, K_{3}}\left\|u_{K_{2}}\right\|_{\infty}\left\|u_{K_{3}}\right\|_{\infty}\left\|G_{K_{3}}\right\|_{\infty} \sum_{K_{0}} \int\left|\partial_{x} u_{K_{0}}\right| \sum_{K_{1}} \sum_{K \leq C K_{1}} K^{s} \tilde{v}_{K}^{*}\left|u_{K_{1}}\right| d x d t .
$$

This is clearly bounded by

$$
C \mathfrak{M}\|u\|_{Y_{s}}^{2} \sum_{K_{0}} \int K_{0}\left|u_{K_{0}}^{*}\right|\left(\sum_{K}\left|\tilde{v}_{K}^{*}\right|^{2}\right)^{1 / 2}\left(\sum_{K_{1}} K_{1}^{2 s}\left|u_{K_{1}}\right|^{2}\right)^{1 / 2} d x d t .
$$

Using the Cauchy-Schwarz inequality, we bound (10-24) by

$$
C \mathfrak{M}\|u\|_{Y_{s}}^{2} \int\left(\sum_{K_{0}} K_{0}^{\varepsilon}\left|u_{K_{0}}^{*}\right|^{2}\right)^{1 / 2}\left(\sum_{K_{0}} K_{0}^{2-\varepsilon} \sum_{K}\left|\tilde{v}_{K}^{*}\right|^{2}\right)^{1 / 2}\left(\sum_{K_{1}} K_{1}^{2 s}\left|u_{K_{1}}\right|^{2}\right)^{1 / 2} d x d t
$$

By Hölder's inequality, (10-25) is majorized by

$$
C \mathfrak{M}\|u\|_{Y_{s}}^{2}\left\|\left(\sum_{K_{0}} K_{0}^{\varepsilon}\left|u_{K_{0}}^{*}\right|^{2}\right)^{1 / 2}\right\|_{4}\left\|\left(\sum_{K_{0}} K_{0}^{2-\varepsilon} \sum_{K}\left|\tilde{v}_{K}^{*}\right|^{2}\right)^{1 / 2}\right\|_{2}\left\|\left(\sum_{K_{1}} K_{1}^{2 s}\left|u_{K_{1}}\right|^{2}\right)^{1 / 2}\right\|_{4},
$$

which is controlled by

$$
C \mathfrak{M}\|u\|_{Y_{s}}^{3}\left\|\partial_{x}^{\varepsilon} u\right\|_{4}\left(\sum_{K_{0}} K_{0}^{2-\varepsilon}\|\tilde{v}\|_{2}^{2}\right)^{1 / 2} \leq C \mathfrak{M}\|u\|_{Y_{s}}^{3}\left\|\partial_{x}^{\varepsilon} u\right\|_{4} \sum_{K_{0}} K_{0}^{-\varepsilon / 2} \leq C \mathfrak{M}\|u\|_{Y_{s}}^{4} .
$$

This finishes the proof of case (10-18).

For case (10-19), let $\tilde{u}$ be defined by

$$
\tilde{u}=\left(\hat{u} \mathbf{1}_{\left|\lambda-n^{3}\right| \geq K_{0}^{2}}\right)^{\vee} .
$$

Then (8-10) can be estimated by

$$
\sum_{K_{2}, K_{3}}\left\|u_{K_{2}}\right\|_{\infty}\left\|u_{K_{3}}\right\|_{\infty}\left\|G_{K_{3}}\right\|_{\infty} \sum_{K_{0}} \int\left|\partial_{x} \tilde{u}_{K_{0}}\right| \sum_{K_{1}} \sum_{K \leq C K_{1}} K^{s} v_{K}^{*}\left|u_{K_{1}}\right| d x d t .
$$

By Schur's test and Hölder's inequality, we control (10-28) by

$$
\sum_{K_{2}, K_{3}}\left\|u_{K_{2}}\right\|_{\infty}\left\|u_{K_{3}}\right\|_{\infty}\left\|G_{K_{3}}\right\|_{\infty} \sum_{K_{0}}\left\|\partial_{x} \tilde{u}_{K_{0}}\right\|_{2}\left\|\left(\sum_{K}\left|v_{K}\right|^{2}\right)^{1 / 2}\right\|_{4}\left\|\left(\sum_{K_{1}} K_{1}^{2 s}\left|u_{K_{1}}\right|^{2}\right)^{1 / 2}\right\|_{4},
$$

which is bounded by

$$
C \mathfrak{M}\|u\|_{Y_{s}}^{3} \sum_{K_{0}}\left\|u_{K_{0}}\right\|_{0,1 / 2} \leq C \mathfrak{M}\|u\|_{Y_{s}}^{4}
$$

This completes the proof of case (10-19). 
For case (10-20), if $j=1$, we dominate (8-10) by

$$
\sum_{K_{2}, K_{3}}\left\|u_{K_{2}}\right\|_{\infty}\left\|u_{K_{3}}\right\|_{\infty}\left\|G_{K_{3}}\right\|_{\infty} \sum_{K_{0}} \int\left|\partial_{x} u_{K_{0}}\right| \sum_{K_{1}} \sum_{K \leq C K_{1}} K^{s} v_{K}^{*}\left|\tilde{u}_{K_{1}}\right| d x d t .
$$

As we did in case (10-19), we bound (10-31) by

$$
C \mathfrak{M}\|u\|_{Y_{s}}^{2} \sum_{K_{0}}\left\|\partial_{x} u_{K_{0}}\right\|_{4}\|v\|_{4}\left\|\left(\sum_{K_{1}} K_{1}^{2 s}\left|\tilde{u}_{K_{1}}\right|^{2}\right)^{1 / 2}\right\|_{2} .
$$

This can be further controlled by

$$
C \mathfrak{M}\|u\|_{Y_{s}}^{3} \sum_{K_{0}} \frac{1}{K_{0}}\left\|\partial_{x} u_{K_{0}}\right\|_{4}\|v\|_{4} \leq \mathfrak{M}\|u\|_{Y_{s}}^{3} \sum_{K_{0}} \frac{1}{K_{0}}\left\|u_{K_{0}}\right\|_{1,1 / 3} \leq C \mathfrak{M}\|u\|_{Y_{s}}^{4},
$$

as desired.

We now consider $j=2$ or $j=3$. Without loss of generality, assume $j=2$. In this case, we estimate $(8-10)$ by

$$
\sum_{K_{3}}\left\|u_{K_{3}}\right\|\left\|G_{K_{3}}\right\|_{\infty} \sum_{K_{0}} \int\left|\partial_{x} u_{K_{0}}\right| \sum_{K_{1}} \sum_{K \leq C K_{1}} K^{s} v_{K}^{*}\left|u_{K_{1}}\right| \sum_{K_{2} \leq C K_{0}}\left|\tilde{u}_{K_{2}}\right| d x d t
$$

which is bounded by

$$
C \mathfrak{M}\|u\|_{Y_{s}} \sum_{K_{0}}\left\|\partial_{x} u_{K_{0}}\right\|_{\infty} \sum_{K_{2} \leq K_{0}}\left\|\tilde{u}_{K_{2}}\right\|_{2}\|v\|_{4}\left\|\left(\sum_{K_{1}} K_{1}^{2 s}\left|u_{K_{1}}\right|^{2}\right)^{1 / 2}\right\|_{4} .
$$

Notice that

$$
\begin{aligned}
\sum_{K_{0}}\left\|\partial_{x} u_{K_{0}}\right\|_{\infty} \sum_{K_{2} \leq K_{0}}\left\|\tilde{u}_{K_{2}}\right\|_{2} & \leq C \sum_{K_{0}} \frac{1}{K_{0}}\left\|\partial_{x} u_{K_{0}}\right\|_{\infty}\|u\|_{Y_{s}} \\
& \leq C \sum_{n} \int|\hat{u}(n, \lambda)| d \lambda\|u\|_{Y_{s}} \leq C\|u\|_{Y_{s}}^{2} .
\end{aligned}
$$

Hence (10-34) is dominated by

$$
C \mathfrak{M}\|u\|_{Y_{s}}^{4}
$$

This completes case (10-20).

We now turn to the most difficult case, (10-21) in case (8-12). We should decompose $G_{K_{3}}$, with respect to the $t$-variable, into Littlewood-Paley multipliers in the same spirit as before. More precisely, for any dyadic number $L$, let $Q_{L}$ be

$$
Q_{L} u(x, t)=\int \psi_{L}(\tau) u(x, t-\tau) d \tau .
$$

Here the Fourier transform of $\psi_{L}$ is a bump function supported on $[-2 L, 2 L]$ and $\widehat{\psi_{L}}(x)=1$ if $x \in[-L, L]$. Let

$$
\Pi_{L} u=Q_{L} u-Q_{L / 2} u
$$


Then $\Pi_{L} u$ gives a Littlewood-Paley multiplier with respect to the time variable $t$. Using this multiplier, we represent

$$
u_{K}=\sum_{L} u_{K, L}
$$

Here $u_{K, L}=\Pi_{L}\left(u_{K}\right)$. We decompose $G_{K_{3}}$ as

$$
\begin{aligned}
G_{K_{3}} & =C+\sum_{L}\left(F_{3}\left(Q_{L} P_{4 K_{3}} u, \ldots, Q_{L} P_{K_{3} / 8} u\right)-F_{3}\left(Q_{L / 2} P_{4 K_{3}} u, \ldots, Q_{L / 2} P_{K_{3} / 8} u\right)\right) \\
& =C+\sum_{j=4,2,1,1 / 2,1 / 4,1 / 8} H_{K_{3}, L} u_{j K_{3}, L},
\end{aligned}
$$

where $H_{K_{3}, L}$ is given by

$$
H_{K_{3}, L}=F_{4}\left(Q_{\ell L} P_{4 K_{3}} u, \ldots, Q_{\ell L} P_{K_{3} / 8} u ; \ell=1, \frac{1}{2}\right) .
$$

Let $\mathfrak{M}_{1}$ be defined by

$$
\mathfrak{M}_{1}=\sup \left\{\left|D^{\alpha} F_{4}\left(u_{1}, \ldots, u_{12}\right)\right|: u_{j} \text { satisfies }\left\|u_{j}\right\|_{Y_{s}} \leq C_{0}\|\phi\|_{H^{s}} \text { for all } j=1, \ldots, 12 ; \alpha\right\} .
$$

Here $D^{\alpha}=\partial_{x_{1}}^{\alpha_{1}} \ldots \partial_{x_{12}}^{\alpha_{12}}$ and $\alpha$ is taken over all tuples $\left(\alpha_{1}, \ldots, \alpha_{12}\right) \in(\mathbb{N} \cup\{0\})^{12}$ with $\sum\left|\alpha_{j}\right| \leq 1 . \mathfrak{M}_{1}$ is a real number because $F_{4} \in C^{1}$.

In order to finish the proof, we need to consider a further three subcases:

$$
\begin{aligned}
L & \leq 2^{10} K_{3}^{3}, \\
2^{10} K_{3}^{3} & <L \leq 2^{-5} K_{0}^{2}, \\
L & >2^{-5} K_{0}^{2} .
\end{aligned}
$$

For the contribution of (10-42), we set

$$
h_{K_{0}, j K_{3}, L}=\left(\widehat{H_{K_{3}, L} u_{j} K_{3}, L} \mathbf{1}_{|\mu| \geq K_{0}^{2}}\right)^{\vee} .
$$

Here $j=4,2,1,1 / 2,1 / 4,1 / 8$. From the definition of $H_{K_{3}, L}$, we get

$$
\left\|h_{K_{0}, j K_{3}, L}\right\|_{4} \leq C \mathfrak{M}_{1}\|\phi\|_{H^{s}} \frac{L}{K_{0}^{2}}\left\|u_{j K_{3}, L}\right\|_{4} .
$$

Then $(8-10)$ is bounded by

$$
\sum_{K_{2}}\left\|u_{K_{2}}\right\|_{\infty} \sum_{K_{0}} \int K_{0} u_{K_{0}}^{*} \sum_{K_{3} \leq C K_{0}^{1 / 2}}\left\|u_{K_{3}}\right\|_{\infty} \sum_{L \leq C K_{3}^{3}}\left|h_{K_{0}, j K_{3}, L}\right| \sum_{K_{1}} \sum_{K \leq C K_{1}} K^{s} v_{K}^{*}\left|u_{K_{1}}\right| d x d t,
$$

which is majorized by

$$
\begin{aligned}
& \sum_{K_{2}}\left\|u_{K_{2}}\right\|_{\infty} \sum_{K_{0}} K_{0} \sum_{K_{3} \leq C K_{0}^{1 / 2}}\left\|u_{K_{3}}\right\|_{\infty} \int u_{K_{0}}^{*} \\
& \cdot \sum_{L \leq C K_{3}^{3}}\left|h_{K_{0}, j K_{3}, L}\right|\left(\sum_{K}\left|v_{K}^{*}\right|^{2}\right)^{1 / 2}\left(\sum_{K_{1}} K_{1}^{2 s}\left|u_{K_{1}}\right|^{2}\right)^{1 / 2} d x d t .
\end{aligned}
$$


Using Hölder's inequality with $L^{4}$ norms for the four functions in the integrand, we estimate (10-48) as follows:

$$
\begin{aligned}
C \mathfrak{M}_{1}\|\phi\|_{H^{s}}\|u\|_{Y_{s}}^{2} \sum_{K_{0}} K_{0}\left\|u_{K_{0}}\right\|_{4} \sum_{K_{3} \leq K_{0}^{1 / 2}}\left\|u_{K_{3}}\right\|_{\infty} \sum_{L \leq C K_{3}^{3}} \frac{L}{K_{0}^{2}}\left\|u_{j K_{3}, L}\right\|_{4} & \\
& \leq C \mathfrak{M}_{1}\|\phi\|_{H^{s}}^{2}\|u\|_{Y_{s}}^{3} \sum_{K_{0}} K_{0}^{1 / 2}\left\|u_{K_{0}}\right\|_{0,1 / 3} \\
& \leq C \mathfrak{M}_{1}\|\phi\|_{H^{s}}^{2}\|u\|_{Y_{s}}^{4} .
\end{aligned}
$$

This finishes case (10-42).

For the contribution of (10-43), we bound (8-10) by

$$
\sum_{K_{2}}\left\|u_{K_{2}}\right\|_{\infty} \sum_{K_{3}}\left\|u_{K_{3}}\right\|_{\infty} \int \sum_{K_{0}}\left|\partial_{x} u_{K_{0}}\right| \sum_{2^{10} K_{3}^{3}<L \leq 2^{-10} K_{0}^{2}}\left|h_{K_{0}, j K_{3}, L}\right| \sum_{K_{1}} \sum_{K \leq C K_{1}} K^{s} v_{K}^{*}\left|u_{K_{1}}\right| d x d t
$$

which is dominated by

$$
\begin{aligned}
C\|u\|_{Y_{s}} \sum_{K_{3}}\left\|u_{K_{3}}\right\|_{\infty} \sum_{\substack{\Delta \leq 2^{-10} \\
\Delta \text { dyadic }}} \int \sum_{K_{0}}\left|\partial_{x} u_{K_{0}}\right| \sum_{\substack{2^{10} K_{3}^{3}<L \\
(\Delta / 2) K_{0}^{2}<L \leq \Delta K_{0}^{2}}}\left|h_{K_{0}, j K_{3}, L}\right| \\
\cdot\left(\sum_{K}\left|v_{K}^{*}\right|^{2}\right)^{1 / 2}\left(\sum_{K_{1}} K_{1}^{2 s}\left|u_{K_{1}}\right|^{2}\right)^{1 / 2} d x d t,
\end{aligned}
$$

By the Cauchy-Schwarz inequality, we further estimate (10-51) by

$$
\begin{aligned}
C\|u\|_{Y_{s}} \sum_{K_{3}}\left\|u_{K_{3}}\right\|_{\infty} \sum_{\substack{\Delta \leq 2^{-10} \\
\Delta \text { dyadic }}} \Delta^{-1 / 2} \int \sum_{K_{0}} \frac{\left|\partial_{x} u_{K_{0}}\right|}{K_{0}} \\
\cdot\left(\sum_{\substack{2^{10} K_{3}^{3}<L \\
(\Delta / 2) K_{0}^{2}<L \leq \Delta K_{0}^{2}}} L\left|h_{K_{0}, j K_{3}, L}\right|^{2}\right)^{1 / 2}\left(\sum_{K}\left|v_{K}^{*}\right|^{2}\right)^{1 / 2}\left(\sum_{K_{1}} K_{1}^{2 s}\left|u_{K_{1}}\right|^{2}\right)^{1 / 2} d x d t .
\end{aligned}
$$

Applying Hölder's inequality with an $L^{\infty}$ norm for the first function in the integrand, an $L^{2}$ norm for the second, and $L^{4}$ norms for the last two functions, we then majorize (10-52) by

$$
C\|u\|_{Y_{s}}^{2} \sum_{K_{3}}\left\|u_{K_{3}}\right\|_{\infty} \sum_{\substack{\Delta \leq 2^{-10} \\ \Delta \text { dyadic }}} \Delta^{-1 / 2} \sum_{K_{0}} \frac{\left\|\partial_{x} u_{K_{0}}\right\|_{\infty}}{K_{0}}\left\|\left(\sum_{\substack{2^{10} K_{3}^{3}<L \\(\Delta / 2) K_{0}^{2}<L \leq \Delta K_{0}^{2}}} L\left|h_{K_{0}, j K_{3}, L}\right|^{2}\right)^{1 / 2}\right\|_{2} .
$$

Notice that if $L \sim \Delta K_{0}^{2}$,

$$
\left\|h_{K_{0}, j K_{3}, L}\right\|_{2} \leq C \mathfrak{M}_{1}\|\phi\|_{H^{s}} \Delta\left\|u_{j K_{3}, L}\right\|_{2}
$$


Thus we have

$$
\begin{aligned}
\left\|\left(\sum_{\substack{2^{10} K_{3}^{3}<L \\
(\Delta / 2) K_{0}^{2}<L \leq \Delta K_{0}^{2}}} L\left|h_{K_{0}, j K_{3}, L}\right|^{2}\right)^{1 / 2}\right\|_{2} & \leq C \mathfrak{M}_{1}\|\phi\|_{H^{s}} \Delta\left(\sum_{\substack{2^{10} K_{3}^{3}<L \\
(\Delta / 2) K_{0}^{2}<L \leq \Delta K_{0}^{2}}} L\left\|u_{j K_{3}, L}\right\|_{2}^{2}\right)^{1 / 2} \\
& \leq C \mathfrak{M}_{1}\|\phi\|_{H^{s}} \Delta\left\|u_{j K_{3}}\right\|_{0,1 / 2} \\
& \leq C \mathfrak{M}_{1}\|\phi\|_{H^{s}}^{2} \Delta .
\end{aligned}
$$

From (10-55), (10-53) is bounded by

$$
C \mathfrak{M}_{1}\|\phi\|_{H^{s}}^{2}\|u\|_{Y_{s}}^{2} \sum_{K_{3}}\left\|u_{K_{3}}\right\|_{\infty} \sum_{\substack{\Delta \leq 2^{-10} \\ \Delta \text { dyadic }}} \Delta^{1 / 2} \sum_{K_{0}} \frac{\left\|\partial_{x} u_{K_{0}}\right\|_{\infty}}{K_{0}}
$$

which is clearly majorized by

$$
C \mathfrak{M}_{1}\|\phi\|_{H^{s}}^{2}\|u\|_{Y_{s}}^{4}
$$

This finishes case (10-43).

For the contribution of (10-44), we estimate (8-10) by

$$
\sum_{K_{2}}\left\|u_{K_{2}}\right\|_{\infty} \sum_{K_{3}}\left\|u_{K_{3}}\right\|_{\infty} \int \sum_{K_{0}}\left|\partial_{x} u_{K_{0}}\right| \sum_{L>2^{-5} K_{0}^{2}}\left|h_{K_{0}, j K_{3}, L}\right| \sum_{K_{1}} \sum_{K \leq C K_{1}} K^{s} v_{K}^{*}\left|u_{K_{1}}\right| d x d t
$$

which is bounded by

$$
\begin{aligned}
& \sum_{K_{2}}\left\|u_{K_{2}}\right\|_{\infty} \sum_{K_{3}}\left\|u_{K_{3}}\right\|_{\infty} \int\left(\sum_{K_{0}} \frac{\left|\partial_{x} u_{K_{0}}\right|^{2}}{K_{0}^{2}}\right)^{1 / 2} \\
& \cdot\left(\sum_{L>2^{-5} K_{0}^{2}} L\left|h_{K_{0}, j K_{3}, L}\right|^{2}\right)^{1 / 2}\left(\sum_{K}\left|v_{K}^{*}\right|^{2}\right)^{1 / 2}\left(\sum_{K_{1}} K_{1}^{2 s}\left|u_{K_{1}}\right|^{2}\right)^{1 / 2} d x d t .
\end{aligned}
$$

Applying Hölder's inequality, we further have

$$
\begin{aligned}
(10-59) & \leq C \mathfrak{M}_{1}\|u\|_{Y_{s}}^{2} \sum_{K_{3}}\left\|u_{K_{3}}\right\|_{\infty} \sum_{K_{0}} \frac{\left\|\partial_{x} u_{K_{0}}\right\|_{\infty}}{K_{0}}\left(\sum_{L>2^{-5} K_{0}^{2}} L\left\|u_{j K_{3}, L}\right\|_{2}^{2}\right)^{1 / 2} \\
& \leq C \mathfrak{M}_{1}\|u\|_{Y_{s}}^{2} \sum_{K_{3}}\left\|u_{K_{3}}\right\|_{\infty} \sum_{K_{0}} \frac{\left\|\partial_{x} u_{K_{0}}\right\|_{\infty}}{K_{0}}\left\|u_{j K_{3}}\right\|_{0,1 / 2} .
\end{aligned}
$$

This is clearly majorized by

$$
C \mathfrak{M}_{1}\|\phi\|_{H_{s}}\|u\|_{Y_{s}}^{4}
$$

Hence we complete case (10-44). 


\section{Proof of case (8-13)}

In this case, it suffices to consider the following subcases:

$$
\begin{aligned}
& M \geq 2^{-10} K_{0}^{2 / 3}, \\
& M<2^{-10} K_{0}^{2 / 3} \quad \text { and } \quad K_{2}^{2} K_{3} \geq 2^{-10} K_{0}^{2}, \\
& M<2^{-10} K_{0}^{2 / 3} \quad \text { and } \quad K_{2}^{2} M \geq 2^{-10} K_{0}^{2}, \\
& M<2^{-10} K_{0}^{2 / 3}, \quad K_{2}^{2} K_{3}<2^{-10} K_{0}^{2} \quad \text { and } \quad K_{2}^{2} M<2^{-10} K_{0}^{2} .
\end{aligned}
$$

For case (11-1), notice that we have

$$
K \leq C M^{3 / 2} .
$$

Hence we estimate (8-10) by

$$
\int \sum_{K_{1} \geq K_{2} \geq K_{3}}\left|u_{K_{1}}\right|\left|u_{K_{2}}\right|\left|u_{K_{3}}\right| \sum_{M} \sum_{K \leq C M^{3 / 2}} K^{s} v_{K}^{*} \sum_{K_{0} \leq C M^{3 / 2}} K_{0} u_{K_{0}}^{*}\left|g_{K_{3}, M}\right| d x d t,
$$

which is bounded by

$$
\int \sum_{K_{1} \geq K_{2} \geq K_{3}}\left|u_{K_{1}}\right|\left|u_{K_{2}}\right|\left|u_{K_{3}}\right| \sum_{M} M^{(3 / 2)(1-s)}\left|g_{K_{3}, M}\right| \sum_{K \leq C M^{3 / 2}} K^{s} v_{K}^{*}\left(\sum_{K_{0}} K_{0}^{2 s}\left|u_{K_{0}}^{*}\right|^{2}\right)^{1 / 2} d x d t,
$$

since $1 / 2<s<1$. Applying Schur's test, we estimate (11-7) by

$$
\int \sum_{K_{1} \geq K_{2} \geq K_{3}}\left|u_{K_{1}}\right|\left|u_{K_{2}}\right|\left|u_{K_{3}}\right|\left(\sum_{M} M^{3}\left|g_{K_{3}, M}\right|^{2}\right)^{1 / 2}\left(\sum_{K}\left|v_{K}^{*}\right|^{2}\right)^{1 / 2}\left(\sum_{K_{0}} K_{0}^{2 s}\left|u_{K_{0}}^{*}\right|^{2}\right)^{1 / 2} d x d t .
$$

By Hölder's inequality and $s>1 / 2,(11-8)$ is majorized by

$$
\begin{aligned}
C \sum_{K_{1} \geq K_{2} \geq K_{3}}\left\|\partial_{x}^{3 / 2} G_{K_{3}}\right\|_{\infty}\left(\prod_{j=1}^{3}\left\|u_{K_{j}}\right\|_{6+}\right)\left\|\left(\sum_{K}\left|v_{K}\right|^{2}\right)^{1 / 2}\right\|\left\|\left(\sum_{K_{0}} K_{0}^{2 s}\left|u_{K_{0}}^{*}\right|^{2}\right)^{1 / 2}\right\| \\
\leq C \mathfrak{M}\left(\|\phi\|_{H^{s}}+\|\phi\|_{H^{s}}^{2}\right)\|u\|_{Y_{s}} \sum_{K_{1} \geq K_{3} \geq K_{3}} K_{3}^{3 / 2} \prod_{j=1}^{3}\left\|u_{K_{j}}\right\|_{6+} \\
\leq C \mathfrak{M}\left(\|\phi\|_{H^{s}}+\|\phi\|_{H^{s}}^{2}\right)\|u\|_{Y_{s}} \prod_{j=1}^{3} \sum_{K_{j}} K_{j}^{1 / 2}\left\|u_{K_{j}}\right\|_{0+, 1 / 2} \\
\leq C \mathfrak{M}\left(\|\phi\|_{H^{s}}+\|\phi\|_{H^{s}}^{2}\right)\|u\|_{Y_{s}}^{4} .
\end{aligned}
$$

This finishes case (11-1).

For case (11-2), observe that, in this case,

$$
K_{0} \leq C K_{1}^{1 / 2} K_{2}^{1 / 2} K_{3}^{1 / 2} .
$$


We estimate (8-10) by

$$
\int \sum_{K_{1} \geq K_{2} \geq K_{3}}\left|u_{K_{1}}\right|\left|u_{K_{2}}\right|\left|u_{K_{3}}\right| \sum_{K \leq C K_{0}} K^{s} v_{K}^{*} \sum_{K_{0} \leq C\left(K_{1} K_{2} K_{3}\right)^{1 / 2}} K_{0} u_{K_{0}}^{*}\left\|G_{K_{3}}\right\|_{\infty} d x d t
$$

which is bounded by

$$
C \mathfrak{M} \int\left(\sum_{K}\left|v_{K}^{*}\right|^{2}\right)^{1 / 2}\left(\sum_{K_{0}} K_{0}^{2 s}\left|u_{K_{0}}^{*}\right|^{2}\right)^{1 / 2} \prod_{j=1}^{3} \sum_{K_{j}} K_{j}^{1 / 2}\left|u_{K_{j}}\right| d x d t .
$$

Using Hölder's inequality with $L^{4}$ norms for the first two functions and $L^{6}$ norms for the last three functions in the integrand, we obtain

$$
C \mathfrak{M}\|u\|_{Y_{s}} \prod_{j=1}^{3}\left\|\sum_{K_{j}} K_{j}^{1 / 2}\left|u_{K_{j}}\right|\right\|_{6} \leq C \mathfrak{M}\|u\|_{Y_{s}}^{4} .
$$

This completes case (11-2).

For case (11-3) we have

$$
K_{0} \leq C K_{1}^{1 / 2} K_{2}^{1 / 2} M^{1 / 2}
$$

Hence we dominate (8-10) by

$$
\int \sum_{K_{1} \geq K_{2} \geq K_{3}}\left|u_{K_{1}}\right|\left|u_{K_{2}}\right|\left|u_{K_{3}}\right| \sum_{M}\left|g_{K_{3}, M}\right| \sum_{K \leq C K_{0}} K^{s} v_{K}^{*} \sum_{K_{0} \leq C\left(K_{1} K_{2} M\right)^{1 / 2}} K_{0} u_{K_{0}}^{*} d x d t
$$

which is bounded by

$$
\begin{aligned}
C \sum_{K_{3}} \int\left(\sum_{K}\left|v_{K}^{*}\right|^{2}\right)^{1 / 2}\left(\sum_{K_{0}} K_{0}^{2 s}\left|u_{K_{0}}^{*}\right|^{2}\right)^{1 / 2}\left|u_{K_{3}}\right| & \cdot\left(\sum_{M} M\left|g_{K_{3}, M}\right|^{2}\right)^{1 / 2} \prod_{j=1}^{2} \sum_{K_{j}} K_{j}^{1 / 2}\left|u_{K_{j}}\right| d x d t .
\end{aligned}
$$

Using Hölder's inequality with $L^{4}$ norms for the first two functions, $L^{6}$ norms for the third, an $L^{p}$ norm with $p$ very large for the fourth, and $L^{6+}$ for the last two functions in the integrand, we obtain

$$
C\|u\|_{Y_{s}} \prod_{j=1}^{2}\left\|\sum_{K_{j}} K_{j}^{1 / 2}\left|u_{K_{j}}\right|\right\|_{6+} \sum_{K_{3}}\left\|u_{K_{3}}\right\|_{6}\left\|\partial_{x}^{1 / 2} G_{K_{3}}\right\|_{\infty}
$$

Clearly (11-17) is dominated by

$$
C \mathfrak{M}\|\phi\|_{H^{s}}\|u\|_{Y_{s}}^{3} \sum_{K_{3}} K_{3}^{1 / 2}\left\|u_{K_{3}}\right\|_{6} \leq C \mathfrak{M}\|\phi\|_{H^{s}}\|u\|_{Y_{s}}^{4} .
$$

Hence case (11-3) is done.

For case (11-4) we observe that

$$
M^{2} K_{2} \leq 2^{-10} K_{0}^{2}
$$


In fact, if (11-19) does not hold, then, from (11-4),

$$
M^{2} K_{2}>2^{-10} K_{0}^{2}>K_{2}^{2} M
$$

Thus $M>K_{2}$, which immediately yields

$$
M^{3}>M^{2} K_{2}>2^{-10} K_{0}^{2},
$$

contradicting $M<2^{-10} K_{0}^{2 / 3}$. Hence (11-19) must be true. From (11-19), $K_{2}^{2} K_{3}+K_{2}^{2} M<2^{-9} K_{0}^{2}$, we get

$$
\left|\left(n_{2}+n_{3}+m\right)^{3}-n_{2}^{3}-n_{3}^{3}-m^{3}\right| \leq 2^{-5} K_{0}^{2}
$$

Since $n_{1}+n_{2}+n_{3}+m \neq 0$, from (8-13), (11-4), and (11-20), the crucial arithmetic observation (10-16) yields

$$
\left|n^{3}-n_{0}^{3}-n_{1}^{3}-n_{2}^{3}-n_{3}^{3}-m^{3}\right| \geq 2 K_{0}^{2} .
$$

Hence one of the following statements must be true:

$$
\begin{aligned}
\left|\lambda-n^{3}\right| & \geq K_{0}^{2}, \\
\left|\left(\lambda-\lambda_{1}-\lambda_{2}-\lambda_{3}-\mu\right)-n_{0}^{3}\right| & \geq K_{0}^{2},
\end{aligned}
$$

there exists an $i \in\{1,2,3\}$ such that $\left|\lambda_{i}-n_{i}^{3}\right| \geq K_{0}^{2}$,

$$
|\mu| \geq K_{0}^{2} \text {. }
$$

For case (11-22), we estimate (8-10) by

$$
\sum_{K_{1}, K_{2}, K_{3}}\left\|u_{K_{1}}\right\|_{\infty}\left\|u_{K_{2}}\right\|_{\infty}\left\|u_{K_{3}}\right\|_{\infty}\left\|G_{K_{3}}\right\|_{\infty} \sum_{K_{0}} \int K_{0}\left|u_{K_{0}}^{*}\right|\left|\sum_{K \leq C K_{0}} \partial_{x}^{s} \tilde{v}_{K}\right| d x d t
$$

Then the Cauchy-Schwarz inequality yields

$$
\begin{aligned}
C \mathfrak{M}\|u\|_{Y_{s}}^{3}\left\|\left(\sum_{K_{0}} K_{0}^{2-2 s}\left|\sum_{K \leq C K_{0}} \partial_{x}^{s} \tilde{v}_{K}\right|^{2}\right)^{1 / 2}\right\|\left\|_{2}\right\|\left(\sum_{K_{0}} K_{0}^{2 s}\left|u_{K_{0}}^{*}\right|^{2}\right)^{1 / 2} \|_{2} \\
\leq C \mathfrak{M}\|u\|_{Y_{s}}^{4}\left(\sum_{K_{0}} K_{0}^{2-2 s} \sum_{K \leq C K_{0}}\left\|\partial_{x}^{s} \tilde{v}_{K}\right\|_{2}^{2}\right)^{1 / 2} \leq C \mathfrak{M}\|u\|_{Y_{s}}^{4} .
\end{aligned}
$$

This finishes the proof of case (11-22).

For case (11-23), (8-10) can be estimated by

$$
\sum_{K_{1}, K_{2}, K_{3}}\left\|u_{K_{1}}\right\|_{\infty}\left\|u_{K_{2}}\right\|_{\infty}\left\|u_{K_{3}}\right\|_{\infty}\left\|G_{K_{3}}\right\|_{\infty} \sum_{K_{0}} \int K_{0}\left|\tilde{u}_{K_{0}}^{*}\right| \sum_{K \leq C K_{0}} K^{s} v_{K}^{*} d x d t
$$

By Schur's test and Hölder's inequality, we control (11-28) by

$$
C \mathfrak{M}\|u\|_{Y_{s}}^{3}\left\|\left(\sum_{K}\left|v_{K}^{*}\right|^{2}\right)^{1 / 2}\right\|_{2}\left\|\left(\sum_{K_{0}} K_{0}^{2 s+2}\left|\tilde{u}_{K_{0}}\right|^{2}\right)^{1 / 2}\right\|_{2},
$$


which is clearly bounded by

$$
C \mathfrak{M}\|u\|_{Y_{s}}^{3}\left(\sum_{K_{0}} K_{0}^{2 s}\left\|u_{K_{0}}\right\|_{0,1 / 2}^{2}\right)^{1 / 2} \leq C \mathfrak{M}\|u\|_{Y_{s}}^{4}
$$

This completes the proof of case (11-23).

For case (11-24), without loss of generality, assume $j=1$. We then dominate (8-10) by

$$
\sum_{K_{2}, K_{3}}\left\|u_{K_{2}}\right\|_{\infty}\left\|u_{K_{3}}\right\|_{\infty}\left\|G_{K_{3}}\right\|_{\infty} \sum_{K_{1}} \sum_{K_{0}} \int K_{0}\left|u_{K_{0}}^{*}\right|\left|\tilde{u}_{K_{1}}\right| \sum_{K \leq C K_{0}} K^{s} v_{K}^{*} d x d t
$$

By Hölder's inequality, we bound (11-31) by

$$
\begin{aligned}
\sum_{K_{2}, K_{3}}\left\|u_{K_{2}}\right\|_{\infty}\left\|u_{K_{3}}\right\|_{\infty}\left\|G_{K_{3}}\right\|_{\infty} \sum_{K_{1}} \sum_{K_{0}} \sum_{K \leq C K_{0}} K^{s} K_{0}\left\|u_{K_{0}}\right\|_{4}\left\|\tilde{u}_{K_{1}}\right\|_{2}\left\|v_{K}\right\|_{4} \\
\leq \sum_{K_{2}, K_{3}}\left\|u_{K_{2}}\right\|_{\infty}\left\|u_{K_{3}}\right\|_{\infty}\left\|G_{K_{3}}\right\|_{\infty} \sum_{K_{1}}\left\|u_{K_{1}}\right\|_{0,1 / 2} \sum_{K_{0}} \sum_{K \leq C K_{0}} K^{s}\left\|u_{K_{0}}\right\|_{4}\left\|v_{K}\right\|_{4} .
\end{aligned}
$$

By Schur's test, we dominate (11-32) by

$$
\begin{aligned}
C \mathfrak{M}\|u\|_{Y_{s}}^{2} \sum_{K_{1}}\left\|u_{K_{1}}\right\|_{0,1 / 2}\left(\sum_{K_{0}} K_{0}^{2 s}\left\|u_{K_{0}}\right\|_{4}^{2}\right)^{1 / 2} & \left(\sum_{K}\left\|v_{K}\right\|_{4}^{2}\right)^{1 / 2} \\
& \leq C \mathfrak{M}\|u\|_{Y_{s}}^{3}\left(\sum_{K_{0}} K_{0}^{2 s}\left\|u_{K_{0}}\right\|_{0,1 / 3}^{2}\right)^{1 / 2}\left(\sum_{K}\left\|v_{K}\right\|_{0,1 / 3}^{2}\right)^{1 / 2} \\
& \leq C \mathfrak{M}\|u\|_{Y_{s}}^{4}
\end{aligned}
$$

Hence case (11-24) is done.

In order to finish the proof, as is done in (10-36), we need to consider three further subcases:

$$
\begin{aligned}
L & \leq 2^{10} K_{3}^{3}, \\
2^{10} K_{3}^{3} & <L \leq 2^{-5} K_{0}^{2}, \\
L & >2^{-5} K_{0}^{2} .
\end{aligned}
$$

For the contribution of (11-34), notice that

$$
\left\|h_{K_{0}, j K_{3}, L}\right\|_{6} \leq C \mathfrak{M}_{1}\|\phi\|_{H^{s}} \frac{L}{K_{0}^{2}}\left\|u_{j K_{3}, L}\right\|_{6} .
$$

Here $h_{K_{0}, j K_{3}, L}$ is defined as in (10-45). In this case we also have $K_{3} \leq K_{0}^{2 / 3}$, from

$$
K_{2}^{2} K_{3} \leq 2^{-10} K_{0}^{2}
$$

Then (8-10) is bounded by

$$
\int \sum_{K_{0}} K_{0} u_{K_{0}}^{*} \sum_{K \leq C K_{0}} K^{s} v_{K}^{*} \sum_{\substack{K_{1} \geq K_{2} \geq K_{3} \\ K_{3} \leq K_{0}^{2 / 3}}}\left|u_{K_{1}}\right|\left|u_{K_{2}}\right|\left|u_{K_{3}}\right| \sum_{L \leq C K_{3}^{3}}\left|h_{K_{0}, j K_{3}, L}\right| d x d t
$$


Write (11-38) as

$$
\sum_{\substack{\Delta \text { dyadic } \\ \Delta \leq 1}} \int \sum_{K_{0}} K_{0} u_{K_{0}}^{*} \sum_{K \leq C K_{0}} K^{s} v_{K}^{*} \sum_{\substack{K_{1} \geq K_{2} \geq K_{3} \\ \Delta K_{0}^{2 / 3} / 2<K_{3} \leq \Delta K_{0}^{2 / 3}}}\left|u_{K_{1}}\right|\left|u_{K_{2}}\right|\left|u_{K_{3}}\right| \sum_{L \leq C K_{3}^{3}}\left|h_{K_{0}, j K_{3}, L}\right| d x d t .
$$

Observe that if $\Delta K_{0}^{2 / 3} / 2<K_{3} \leq \Delta K_{0}^{2 / 3}$, we have

$$
K_{0} \leq \Delta^{-3 / 2} K_{1}^{1 / 2} K_{2}^{1 / 2} K_{3}^{1 / 2}
$$

Hence

$$
\begin{aligned}
C\|u\|_{Y_{s}} \sum_{K_{0}} \sum_{K \leq K_{0}} K^{s} \sum_{K_{1}, K_{2}} K_{1}^{1 / 2} K_{2}^{1 / 2} \sum_{\Delta \leq 1} \Delta^{-3 / 2} & \sum_{K_{3} \sim \Delta K_{0}^{2 / 3}} K_{3}^{1 / 2} \\
& \cdot \int u_{K_{0}}^{*} v_{K}^{*}\left|u_{K_{1}}\right|\left|u_{K_{2}}\right| \sum_{L \leq C K_{3}^{3}}\left|h_{K_{0}, j K_{3}, L}\right| d x d t .
\end{aligned}
$$

Applying Hölder's inequality with $L^{4}$ norms for first two functions and $L^{6}$ for the last three, and then using (11-37), we get

$$
\begin{aligned}
& C \mathfrak{M}_{1}\|\phi\|_{H^{s}}\|u\|_{Y_{s}} \sum_{K_{0}} \sum_{K \leq K_{0}} K^{s} \sum_{K_{1}, K_{2}} K_{1}^{1 / 2} K_{2}^{1 / 2} \sum_{\Delta \leq 1} \Delta^{-3 / 2} \\
& \cdot \sum_{K_{3} \sim \Delta K_{0}^{2 / 3}} K_{3}^{1 / 2}\left\|u_{K_{0}}\right\|_{4}\left\|v_{K}^{*}\right\|_{4}\left\|u_{K_{1}}\right\|_{6}\left\|u_{K_{2}}\right\|_{6} \sum_{L \leq C K_{3}^{3}} \frac{L}{K_{0}^{2}}\left\|u_{j K_{3}, L}\right\|_{6},
\end{aligned}
$$

which is bounded by

$$
\begin{gathered}
C \mathfrak{M}_{1}\|\phi\|_{H^{s}}\|u\|_{Y_{s}} \sum_{K_{0}} \sum_{K \leq K_{0}} K^{s} \sum_{\Delta \leq 1} \Delta^{-3 / 2} \sum_{L \leq C \Delta^{3} K_{0}^{2}} \frac{L}{K_{0}^{2}}\left\|u_{K_{0}}\right\|_{4}\left\|v_{K}^{*}\right\|_{4} \\
\cdot \sum_{K_{1}} K_{1}^{1 / 2}\left\|u_{K_{1}}\right\|_{0+, 1 / 2} \sum_{K_{2}} K_{2}^{1 / 2}\left\|u_{K_{2}}\right\|_{0+, 1 / 2} \sum_{K_{3}} K_{3}^{1 / 2}\left\|u_{j K_{3}, L}\right\|_{0+, 1 / 2} \\
\leq C \mathfrak{M}_{1}\|\phi\|_{H^{s}}^{2}\|u\|_{Y_{s}}^{3} \sum_{\Delta \leq 1} \Delta^{3 / 2} \sum_{K_{0}} \sum_{K \leq C K_{0}} K^{s}\left\|u_{K_{0}}\right\|_{4}\left\|v_{K}\right\|_{4} \\
\leq C \mathfrak{M}_{1}\|\phi\|_{H^{s}}^{2}\|u\|_{Y_{s}}^{3}\left(\sum_{K_{0}} K_{0}^{2 s}\left\|u_{K_{0}}\right\|_{0,1 / 3}^{2}\right)^{1 / 2}\left(\sum_{K}\left\|v_{K}\right\|_{0,1 / 3}^{2}\right)^{1 / 2} \\
\leq C \mathfrak{M}_{1}\|\phi\|_{H^{s}}^{2}\|u\|_{Y_{s}}^{4} .
\end{gathered}
$$

This completes case (11-34).

For the contribution of (11-35), we bound (8-10) by

$$
\sum_{K_{1}}\left\|u_{K_{1}}\right\|_{\infty} \sum_{K_{2}}\left\|u_{K_{2}}\right\|_{\infty} \sum_{K_{3}}\left\|u_{K_{3}}\right\|_{\infty} \int \sum_{K_{0}} \sum_{K \leq C K_{0}} K^{s} v_{K}^{*} K_{0} u_{K_{0}}^{*} \sum_{2^{10} K_{3}^{3}<L \leq 2^{-5} K_{0}^{2}}\left|h_{K_{0}, j K_{3}, L}\right| d x d t
$$


which is dominated by

$$
C\|u\|_{Y_{s}}^{2} \sum_{K_{3}}\left\|u_{K_{3}}\right\|_{\infty} \sum_{\substack{\Delta \leq 2^{-5} \\ \Delta \text { dyadic }}} \sum_{K_{0}} \sum_{K \leq C K_{0}} K^{s} \int K_{0} u_{K_{0}}^{*} v_{K}^{*} \sum_{\substack{2^{10} K_{3}^{3}<L \\(\Delta / 2) K_{0}^{2}<L \leq \Delta K_{0}^{2}}}\left|h_{K_{0}, j K_{3}, L}\right| d x d t .
$$

Using the Cauchy-Schwarz inequality, we further estimate (11-45) by

$$
C\|u\|_{Y_{S}}^{2} \sum_{K_{3}}\left\|u_{K_{3}}\right\|_{\infty} \sum_{\substack{\Delta \leq 2^{-5} \\ \Delta \text { dyadic }}} \Delta^{-\frac{1}{2}} \sum_{K_{0}} \sum_{K \leq C K_{0}} K^{s} \int u_{K_{0}}^{*} v_{K}^{*}\left(\sum_{\substack{2^{10} K_{3}^{3}<L \\(\Delta / 2) K_{0}^{2}<L \leq \Delta K_{0}^{2}}} L\left|h_{K_{0}, j K_{3}, L}\right|^{2}\right)^{1 / 2} d x d t
$$

Employing Hölder's inequality with $L^{4}$ norms for the first two functions and an $L^{2}$ for the last one, we bound (11-46) by

$C\|u\|_{Y_{s}}^{2}$

$$
\cdot \sum_{K_{3}}\left\|u_{K_{3}}\right\|_{\infty} \sum_{\substack{\Delta \leq 2^{-10} \\ \Delta \text { dyadic }}} \Delta^{-1 / 2} \sum_{K_{0}} \sum_{K \leq C K_{0}} K^{s}\left\|u_{K_{0}}\right\|_{4}\left\|v_{K}\right\|_{4}\left\|\left(\sum_{\substack{(\Delta / 2) K_{0}^{2}<L \leq \Delta K_{0}^{2} \\ 2^{10} K_{3}^{3}<L}} L\left|h_{K_{0}, j K_{3}, L}\right|^{2}\right)^{1 / 2}\right\|_{2} .
$$

From (10-55), (11-47) is majorized by

$$
\begin{gathered}
C \mathfrak{M}_{1}\|\phi\|_{H^{s}}^{2}\|u\|_{Y_{s}}^{2} \sum_{K_{3}}\left\|u_{K_{3}}\right\|_{\infty} \sum_{\substack{\Delta \leq 2^{-10} \\
\Delta \text { dyadic }}} \Delta^{\frac{1}{2}} \sum_{K_{0}} \sum_{K \leq C K_{0}} K^{s}\left\|u_{K_{0}}\right\|_{4}\left\|v_{K}\right\|_{4} \\
\leq C \mathfrak{M}_{1}\|\phi\|_{H^{s}}^{2}\|u\|_{Y_{s}}^{3}\left(\sum_{K_{0}} K_{0}^{2 s}\left\|u_{K_{0}}\right\|_{0, \frac{1}{3}}^{2}\right)^{1 / 2}\left(\sum_{K}\left\|v_{K}\right\|_{0, \frac{1}{3}}^{2}\right)^{1 / 2} \\
\leq C \mathfrak{M}_{1}\|\phi\|_{H^{s}}^{2}\|u\|_{Y_{s}}^{4}
\end{gathered}
$$

This finishes the proof for case (11-35).

For the contribution of (11-36), we estimate (8-10) by

$$
\sum_{K_{1}, K_{2}}\left\|u_{K_{1}}\right\|_{\infty}\left\|u_{K_{2}}\right\|_{\infty} \sum_{K_{3}}\left\|u_{K_{3}}\right\|_{\infty} \int \sum_{K_{0}} K_{0} u_{K_{0}}^{*} \sum_{L>2^{-5} K_{0}^{2}}\left|h_{K_{0}, j K_{3}, L}\right| \sum_{K \leq C K_{0}} K^{s} v_{K}^{*} d x d t
$$

By the Cauchy-Schwarz inequality, (11-49) is bounded by

$$
\sum_{K_{1}, K_{2}}\left\|u_{K_{1}}\right\|_{\infty}\left\|u_{K_{2}}\right\|_{\infty} \sum_{K_{3}}\left\|u_{K_{3}}\right\|_{\infty} \sum_{K_{0}} \sum_{K \leq C K_{0}} K^{s} \int v_{K}^{*} u_{K_{0}}^{*}\left(\sum_{L>2^{-10} K_{0}^{2}} L\left|h_{K_{0}, j K_{3}, L}\right|^{2}\right)^{1 / 2} d x d t
$$

Employing Hölder's inequality with $L^{4}$ norms for the first two functions and an $L^{2}$ for the last one, we 
dominate (11-50) by

$$
\begin{gathered}
C \mathfrak{M}_{1}\|u\|_{Y_{s}}^{2} \sum_{K_{3}}\left\|u_{K_{3}}\right\|_{\infty} \sum_{K_{0}} \sum_{K \leq C K_{0}} K^{s}\left\|u_{K_{0}}\right\|_{4}\left\|v_{K}\right\|_{4}\left\|\left(\sum_{L>2^{-5} K_{0}^{2}} L\left|u_{j K_{3}, L}\right|^{2}\right)^{1 / 2}\right\|_{2} \\
\leq C \mathfrak{M}_{1}\|u\|_{Y_{s}}^{2} \sum_{K_{3}}\left\|u_{K_{3}}\right\|_{\infty} \sum_{K_{0}} \sum_{K \leq C K_{0}} K^{s}\left\|u_{K_{0}}\right\|_{0,1 / 3}\left\|v_{K}\right\|_{0,1 / 3}\|u\|_{0,1 / 2} \\
\leq C \mathfrak{M}_{1}\|\phi\|_{H^{s}}\|u\|_{Y_{s}}^{4} .
\end{gathered}
$$

Hence we complete case (11-36).

\section{References}

[Bourgain 1993a] J. Bourgain, "Fourier transform restriction phenomena for certain lattice subsets and applications to nonlinear evolution equations, I: Schrödinger equations”, Geom. Funct. Anal. 3:2 (1993), 107-156. MR 95d:35160a Zbl 0787.35097

[Bourgain 1993b] J. Bourgain, "Fourier transform restriction phenomena for certain lattice subsets and applications to nonlinear evolution equations, II: The KdV-equation”, Geom. Funct. Anal. 3:3 (1993), 209-262. MR 95d:35160b Zbl 0787.35098

[Bourgain 1995] J. Bourgain, "On the Cauchy problem for periodic KdV-type equations", pp. 17-86 in Proceedings of the Conference in Honor of Jean-Pierre Kahane (Orsay, 1993), J. Fourier Anal. Appl. Special Issue, CRC Press, Boca Raton, FL, 1995. MR 96m:35270 Zbl 0891.35137

[Colliander et al. 2003] J. Colliander, M. Keel, G. Staffilani, H. Takaoka, and T. Tao, "Sharp global well-posedness for KdV and modified KdV on $\mathbb{R}$ and T", J. Amer. Math. Soc. 16:3 (2003), 705-749. MR 2004c:35352 Zbl 1025.35025

[Colliander et al. 2004] J. Colliander, M. Keel, G. Staffilani, H. Takaoka, and T. Tao, "Multilinear estimates for periodic KdV equations, and applications", J. Funct. Anal. 211:1 (2004), 173-218. MR 2005a:35241 Zbl 1062.35109

[Hua 1965] L. K. Hua, Additive theory of prime numbers, Translations of Mathematical Monographs 13, American Mathematical Society, Providence, RI, 1965. MR 33 \#2614 Zbl 0192.39304

[Kenig et al. 1996] C. E. Kenig, G. Ponce, and L. Vega, "A bilinear estimate with applications to the KdV equation", J. Amer. Math. Soc. 9:2 (1996), 573-603. MR 96k:35159 Zbl 0848.35114

[Montgomery 1994] H. L. Montgomery, Ten lectures on the interface between analytic number theory and harmonic analysis, CBMS Regional Conference Series in Mathematics 84, American Mathematical Society, Providence, RI, 1994. MR 96i:11002 Zbl 0814.11001

Received 13 Oct 2011. Revised 16 Jun 2012. Accepted 21 Jul 2012.

YI HU: yihu1@illinois.edu

Department of Mathematics, University of Illinois at Urbana-Champaign, 1409 W. Green Street, Urbana, IL 61801, United States Current address: Department of Mathematical Sciences, Georgia Southern University, 65 Georgia Ave. Room 3008, Statesboro, GA 30458, United States

XiAOCHUN LI: xcli@math.uiuc . edu

Department of Mathematics, University of Illinois at Urbana-Champaign, 1409 W. Green Street, Urbana, IL 61801, United States 


\title{
Analysis \& PDE
}

\author{
msp.org/apde
}

\section{EDITORS}

EDITOR-IN-CHIEF

\author{
Maciej Zworski \\ zworski@math.berkeley.edu \\ University of California \\ Berkeley, USA
}

BOARD OF EDITORS

\begin{abstract}
Michael Aizenman
Luis A. Caffarelli

Michael Christ

Ursula Hamenstaedt

Vaughan Jones

Izabella Laba

László Lempert

Frank Merle

Werner Müller

Gilles Pisier

Igor Rodnianski

Sylvia Serfaty

Terence Tao

Gunther Uhlmann

Dan Virgil Voiculescu

Princeton University, USA aizenman@math.princeton.edu

University of Texas, USA caffarel@math.utexas.edu

University of California, Berkeley, USA mchrist@math.berkeley.edu

Universität Bonn, Germany ursula@math.uni-bonn.de

University of California, Berkeley, USA vfr@math.berkeley.edu

University of British Columbia, Canada ilaba@math.ubc.ca

Purdue University, USA lempert@math.purdue.edu

Université de Cergy-Pontoise, France Frank.Merle@u-cergy.fr

Universität Bonn, Germany mueller@math.uni-bonn.de

Texas A\&M University, and Paris 6 pisier@math.tamu.edu

Princeton University, USA irod@math.princeton.edu

New York University, USA serfaty@cims.nyu.edu

University of California, Los Angeles, USA tao@math.ucla.edu

University of Washington, USA gunther@math.washington.edu

University of California, Berkeley, USA dvv@math.berkeley.edu
\end{abstract}

Nicolas Burq Université Paris-Sud 11, France nicolas.burq@math.u-psud.fr

Sun-Yung Alice Chang Princeton University, USA chang@math.princeton.edu

Charles Fefferman Princeton University, USA cf@math.princeton.edu

Nigel Higson Pennsylvania State Univesity, USA higson@math.psu.edu

Herbert Koch Universität Bonn, Germany koch@math.uni-bonn.de

Gilles Lebeau Université de Nice Sophia Antipolis, France lebeau@unice.fr

Richard B. Melrose Massachussets Institute of Technology, USA rbm@math.mit.edu

William Minicozzi II

Johns Hopkins University, USA minicozz@math.jhu.edu

Yuval Peres University of California, Berkeley, USA peres@stat.berkeley.edu

Tristan Rivière ETH, Switzerland riviere@math.ethz.ch

Wilhelm Schlag University of Chicago, USA schlag@math.uchicago.edu

Yum-Tong Siu Harvard University, USA siu@math.harvard.edu

Michael E. Taylor Univ. of North Carolina, Chapel Hill, USA met@math.unc.edu

András Vasy Stanford University, USA andras@math.stanford.edu

Steven Zelditch Northwestern University, USA zelditch@math.northwestern.edu

PRODUCTION

production@msp.org

Silvio Levy, Scientific Editor

See inside back cover or msp.org/apde for submission instructions.

The subscription price for 2013 is US $\$ 160 /$ year for the electronic version, and \$310/year (+\$35, if shipping outside the US) for print and electronic. Subscriptions, requests for back issues from the last three years and changes of subscribers address should be sent to MSP.

Analysis \& PDE (ISSN 1948-206X electronic, 2157-5045 printed) at Mathematical Sciences Publishers, 798 Evans Hall \#3840, c/o University of California, Berkeley, CA 94720-3840, is published continuously online. Periodical rate postage paid at Berkeley, CA 94704, and additional mailing offices.

APDE peer review and production are managed by EditFLow ${ }^{\circledR}$ from Mathematical Sciences Publishers.

\section{PUBLISHED BY}

mathematical sciences publishers

nonprofit scientific publishing

http://msp.org/

(C) 2013 Mathematical Sciences Publishers 


\section{ANALYSIS \& PDE}

\section{Volume $6 \quad$ No. $4 \quad 2013$}

Cauchy problem for ultrasound-modulated EIT

GUILlAUME BAL

Sharp weighted bounds involving $A_{\infty}$

TUOMAS HyTÖNEN and CARLOS PÉREZ

Periodicity of the spectrum in dimension one

Alex Iosevich and Mihal N. Kolountzakis

A codimension-two stable manifold of near soliton equivariant wave maps

IOAN BEJENARU, JOACHIM KRIEGER and DANIEL TATARU

Discrete Fourier restriction associated with KdV equations

YI HU and XIAOCHUN LI

Restriction and spectral multiplier theorems on asymptotically conic manifolds

Colin Guillarmou, Andrew Hassell and Adam SiKora

Homogenization of Neumann boundary data with fully nonlinear operator

Sunhi Choi, Inwon C. Kim and KI-Ahm LeE

Long-time asymptotics for two-dimensional exterior flows with small circulation at infinity

973

THIERRY GALLAY and YASUNORI MAEKAWA

Second order stability for the Monge-Ampère equation and strong Sobolev convergence of 993 optimal transport maps

Guido De PhilipPis and Alessio Figalli 